\title{
Megascolex (Perichaeta) diffringens Baird, 1869 and Pheretima pingi Stephenson, 1925 types compared to the Amynthas corticis (Kinberg, 1867) and A. carnosus (Goto \& Hatai, 1899) species-groups (Oligochaeta: Megadrilacea: Megascolecidae)
}

\author{
Robert J. Blakemore* \\ Biodiversity Laboratory, College of Natural Science, Hanyang University, Seoul 133-791, Korea \\ *Correspondent: rob.blakemore@gmail.com
}

\begin{abstract}
Amynthas diffringens (Baird, 1869) is deemed valid yet comparable to A. corticis (Kinberg, 1867) and to A. nipponicus (Beddard, 1893) or A. peregrinus (Fletcher, 1896). Its syntypes tend to more genital markings than usual as shown for variable specimens from Japan, Korea, and Australasia that comply genetically with either of two A. corticis spp. groups. A. diffringens type-locality was UK hothouses yet closest specimens appear in part of A. heteropodus (Goto \& Hatai, 1899) (=A. corticis) from Japan. Japanese Amynthas divergens (Michaelsen, 1892) is restored based on its serrate intestinal caeca. Meanwhile, the lectotype of Amynthas pingi (Stephenson, 1925) is deemed a synonym of A. carnosus (Goto \& Hatai, 1899) compliant with Kobayashi's (1936) types III \& II. Erstwhile A. pingi synonym A. fornicatus (Gates, 1925) is again included, but A. hongkongensis (Michaelsen, 1910) and A. chungkingensis (Chen, 1936) are provisionally retained whilst A. carnosa lichuanensis Wang \& Qiu, 2005 is given separate species status. A new Korean taxon is proposed as A. carnosus naribunji sub-sp. nov. and two replacement names are provided: A. zhuya nom. nov. for homonym A. montanus Qiu \& Sun, 2012 from Hainan and A. yizhou for A. carnosus sensu Shen et al., 2005 from Taiwan. No attempt is made to fully resolve numerous Japanese synonyms of A. carnosus [eg Ishizuka's (2001) invista, subterranea, subalpina, umbrosa, mutabilis, nubicola, plus A. nonmonticolus Blakemore, 2010] nor Korean synonyms [eg kyamikia Kobayashi, 1934, monstrifera Kobayashi, 1936 and murayamai Kobayashi, 1938, sangyeoli, youngtai (with segments miscounted), kimhaeiensis, sinsiensis and baemsagolensis - all names by Hong \& James (2001) plus ?A. sangumburi Hong \& Kim, 2002 (its segments miscounted too)] also A. fuscus Qiu \& Sun, 2012 from Hainan and Taiwanese monsoonus James et al., 2005 plus A. penpuensis Shen et al., 2003 and A. taiwumontis Shen et al., 2013 syns. nov.. All synonyms remain in the currently defined A. carnosus pending full revisons although several may eventually comply with parts of prior A. corticis s. lato.
\end{abstract}

Keywords: Asian taxonomic, Earthworms, pheretimoid synonyms, species valid

(C) 2013 National Institute of Biological Resources

DOI: 10.12651/JSR.2013.2.2.099

\section{INTRODUCTION}

The pheretimoids are the largest megascolecid group with $\sim 970$ valid names from ca. 1,350 nominal taxa (Blakemore, 2008). Further names are continually added despite several controversies and chronic confusion existing yet for certain groups. This paper aims to contribute to resolution via inspection of historical types.

Amynthas corticis (Kinberg, 1867) is the most commonly recorded cosmopolitan pheretimoid (Blakemore, 2012d). This is due partly to its earlier naming, to its partheno- genetic variability allowing a wide description unconstrained by details and genetics from the immature type and thus many probable misidentifications. Prior to the synonymy of A. corticis by Easton (1981) (taxon misspelt "corticus"), most specimens would have been placed under the second earliest name: Pheretima diffringens (Baird, 1869). Here the syntypes of this species are inspected and the type-locality searched in order to help resolve the boundaries of both of these and of related taxa.

During their long histories, Pheretima divergens (Michaelsen, 1892) from Japan (Berlin type 2116 lost), has been mutually confused with both A. corticis/diffringens 
Table 1. Ohfuchi's (1937) characters of some of his species (in same order he used)

\begin{tabular}{lllccc}
\hline \multicolumn{1}{c}{ Species } & GM anterior & GM posterior & Intestinal caeca & Spermathecal pores & Diverticular stalk \\
\hline P. heteropoda & Y or N & N & Smooth & Small & Medium \\
P. carnosa* & Y $($ or N)* & Y $($ or N)* & Smooth & Large & Medium \\
$P$. oyamai & N & Y & Indented & Small & Absent \\
P. divergens & Y or N & Y or N & Serrate & Small & Variable \\
$P$. nipponica & Y or N & N & Smooth & Variable \\
\hline
\end{tabular}

*Kobayashi (1936) had shown P. carnosa to most commonly lack GMs.

and, despite being earlier taxa, with P. carnosa (Goto \& Hatai, 1899). However, Ohfuchi (1937) seemingly resolved carnosa and divergens as summarized in Table 1.

Ohfuchi (1937) failed to adequately separate $P$. heteropoda (Goto \& Hatai, 1899) from prior P. nipponica (Beddard, 1893) - possibly to avoid upsetting his mentor Hatai; he also seemed unaware of $P$. diffringens and no one at that time considered $A$. corticis.

Another chronically confused taxon is Amynthas pingi (Stephenson, 1925) that was erected and maintained on the assumption that Goto \& Hatai's (1899) prior but poorly described $P$. carnosa had spermathecal pores in 5/6/ $7 / 8$ and markings in $7 \& 8$, whereas it was clearly demonstrated by Kobayashi (1936) and Ohfuchi (1937) that the actual situations were 5/6/7/8/9 and $8 \& 9$, respectively (see Blakemore, 2012a). Subsequent authors misinterpreted or overlooked the redefined Pheretima carnosa and the description of $P$. ping $i$ was later mixed with parts of the A. corticis species-complex, most notably by G.E. Gates (vide infra) who was most contradictory and overconfident in his own assumptions.

Chen (1936: 274) when describing $P$. pingi chungkingensis (that now provisionally merits elevation to species level as per Blakemore, 2012a) remarked that pingi was probably a synonym of carnosa as independently concluded by Kobayashi (1936). Both were ignored by Gates (1939) who, although claiming to have inspected $P$. ping $i$ type, made several false statements. Moreover, Gates (1939: 436) doubted Chen (1936: 298) saying his $P$. fornicata Gates, 1925 "is probably identical with $P$. pingi Steph.", perhaps because it lacks genital markings; nevertheless, there is similarity that merits further investigation. Kobayashi (1936) included $P$. pingi in synonymy of his corrected $P$. carnosa but Blakemore (2012a; 2013a) provisionally retained it separately pending inspection of the type in the British Museum - this now accomplished.

Types help solve chronic taxonomic issues. The current report redescribes Pheretima pingi type (inspected but badly misdescribed by Gates, 1939) allowing controversy of its relationship to both A. carnosus and A. corticis to be resolved. A neotype was recently designated for Amynthas carnosus (Goto \& Hatai, 1889) and its synonyms revised (Blakemore, 2012a-d; et al., 2012; 2013a) where it was confirmed as well represented in Japan and
Korea. Relationship of A. carnosus s. stricto contrasts genetically with Korean material described as A. carnosus roki Blakemore, 2013 (in Blakemore and Lee, 2013) and with an anomalous specimen from Ulleungdo herein considered a new taxon (cf Blakemore, 2013a). Sub-species Amynthas carnosus lichuanensis Wang \& Qiu, 2005 from China was elevated to separate species status in Blakemore and Lee (2013).

\section{Materials ANd Methods}

The taxonomic determination follows the style, systematics, methodology and conventions in Blakemore (2008; 2012d). Small tissue samples from non-essential posterior segments for DNA analyses and (CO1) barcoding as per Blakemore et al. (2010) yielded variable results, especially on older type material. Some of these DNA data are presented in an Appendix. Discussion is confined to remarks after species' descriptions. Abbreviations are GMs - genital markings; rhs, lhs - right, left-handsidep; cf - compare. A "?" indicates some taxonomic uncertainty.

\section{SuRvey Results}

Collections by the author from Japan, New Zealand and Korea (including Jeju) are presented in the taxonomic section below. A brief survey was undertaken at "Y Plas" homestead at Machynlleth, North Wales - the type-locality of Amynthas diffringens - on $22^{\text {nd }}$ June, 2013. The glasshouse nursery no longer exists and searches of likely 'hot-spots' at this UK public Heritage Trust house failed to reveal target specimens. However, several other earthworms were found showing a remarkable diversity, as is obtainable when directed collection is combined with competent taxonomy. The fourteen plus species, deposited in London Natural History Museum, some for DNA analysis, were:

1/. Allolobophora chlorotica (Savigny, 1826); 2/. Aporrectodea caliginosa (Savigny, 1826); 3/. A. longa (Ude, 1885); 4/. A. rosea (Savigny, 1826); 5/. Dendrobaena octaedra (Savigny, 1826); 6/. Dendrodrilus rubidus (Sav- 
igny, 1826); 7/. Eisenia fetida (Savigny, 1826); 8/. Eiseniella tetraedra (Savigny, 1826); 9/. Lumbricus festivus (Savigny, 1826); 10/. L. rubellus Hoffmeister, 1843; 11/. L. terrestris Linnaeus, 1758; 12/. Octolasion cyaneum (Savigny, 1826); 13/. Satchellius mammalis (Savigny, 1826); 14/. Allolobophoridiella eiseni (Levinson, 1884) ( $9^{\text {th }}$ June, 2012 ex patio at Alkington Rd., Whitchurch, Shropshire); 15/. Enchytraeus spp. noted but as unimportant not kept. This number of species found in such a restricted area in such a short period possibly qualifies Y Plas as one of the most diverse UK habitats, after Kew Gardens of course.

\section{Taxonomic Results and Discussion}

Specimens of taxa under consideration are analysed genetically in Fig. 1 and an Appendix.

Annelida Lamarck, 1802

Oligochaeta Grube, 1850

Megadrilacea Benham, 1890

Megascolecidae Rosa, 1891 sensu Blakemore, 2000

Amynthas Kinberg, 1867
Amynthas carnosus carnosus (Goto \& Hatai, 1899)

(Figs. 2-3)

Delailed by Kobayashi (1936), Ohfuchi (1937) and Blakemore (2012a; 2013a; 2013b) (cf A. pingi described below). DNA data (Fig. 1) indicates a new sub-species as next described.

Amynthas carnosus naribunji Blakemore sub-sp. nov. ?Amynthas pingi: Blakemore, 2013a: 60, figs. 4, 5; Blakemore and Lee, 2013.

(Fig. 1)

Material examined. NIBRIV261262 an anomalous mature specimen from Naribunji, Ulleung-do $\left(37^{\circ} 30^{\prime} \mathrm{N}\right.$ $\left.130^{\circ} 52^{\prime} \mathrm{E}\right)$; dissected and providing DNA sample w54 (Appendix).

Etymology. Named for its type locality on Ulleungdo, although there is no implication that it is an endemic, more likely it is introduced as with all the island's other earthworms.

Description. Size $215 \mathrm{~mm}$. Colour a darker brown pigmentation dorsally. First dorsal pore 12/13. Spermathecal pores in 5/6/7/8/9 each with pre-intersegmental hemispherical arcs (spermathecal papillae) anterior to interseg-

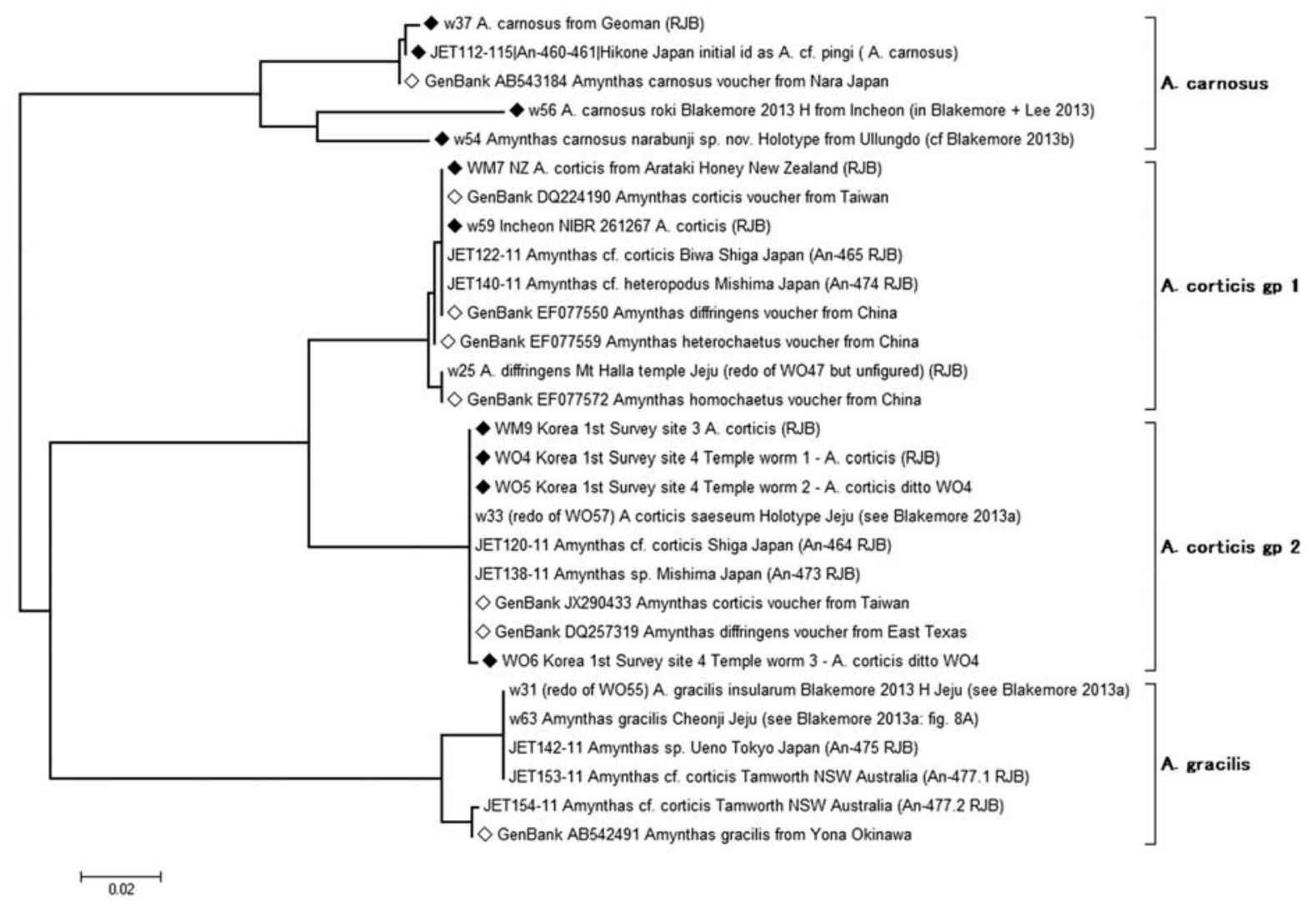

Fig. 1. Phylotree from MEGA5 program Maximum Likelihood defaults - sample codes explained in the Appendix; $\bullet$ samples have figured specimens; $\diamond$ - ex GenBank. Appears to show significant genetic difference between specimens superficially compliant with broad A. corticis definition as discussed below that taxon herein. Synonym priority for either Gp 1 or Gp 2 is currently unresolved. 

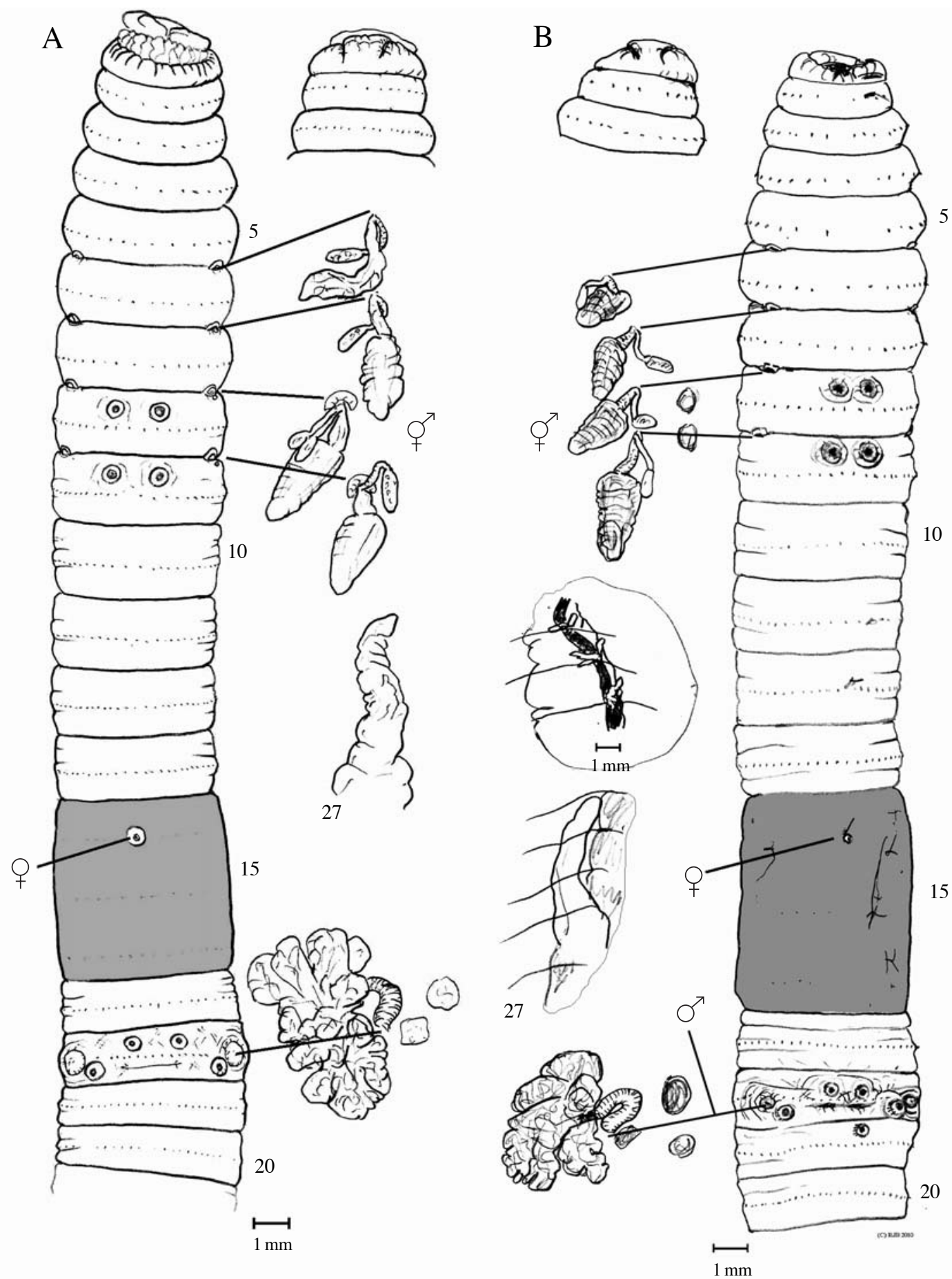

Fig. 2. A. carnosus - A. Korean specimen from Geoman (NIBR IV261234 - DNA w37). B. Japanese neotype of A. carnosus (Tokyo An435 from Blakemore, 2012a). These two specimens are almost identical morphologically allowing genetic comparison (Fig. 1).

ments. Male pores superficial on 18 with disc-like GMs posterio-median to male pores. Setae about 17-18 between male pores. Septa 8/9/10 aborted. Last hearts in 13. Septal glands pronounced from 15 on dorsal blood vessel. Intestinal caeca simple.
Remarks. Otherwise complying within broad description of A. carnosus, the current specimen was originally noted by Blakemore (2013a: 60) in a provisionally restored A. pingi (Stephenson, 1925), the next available synonym of A. carnosus then A. kyamikius (Kobayashi, 1934) 


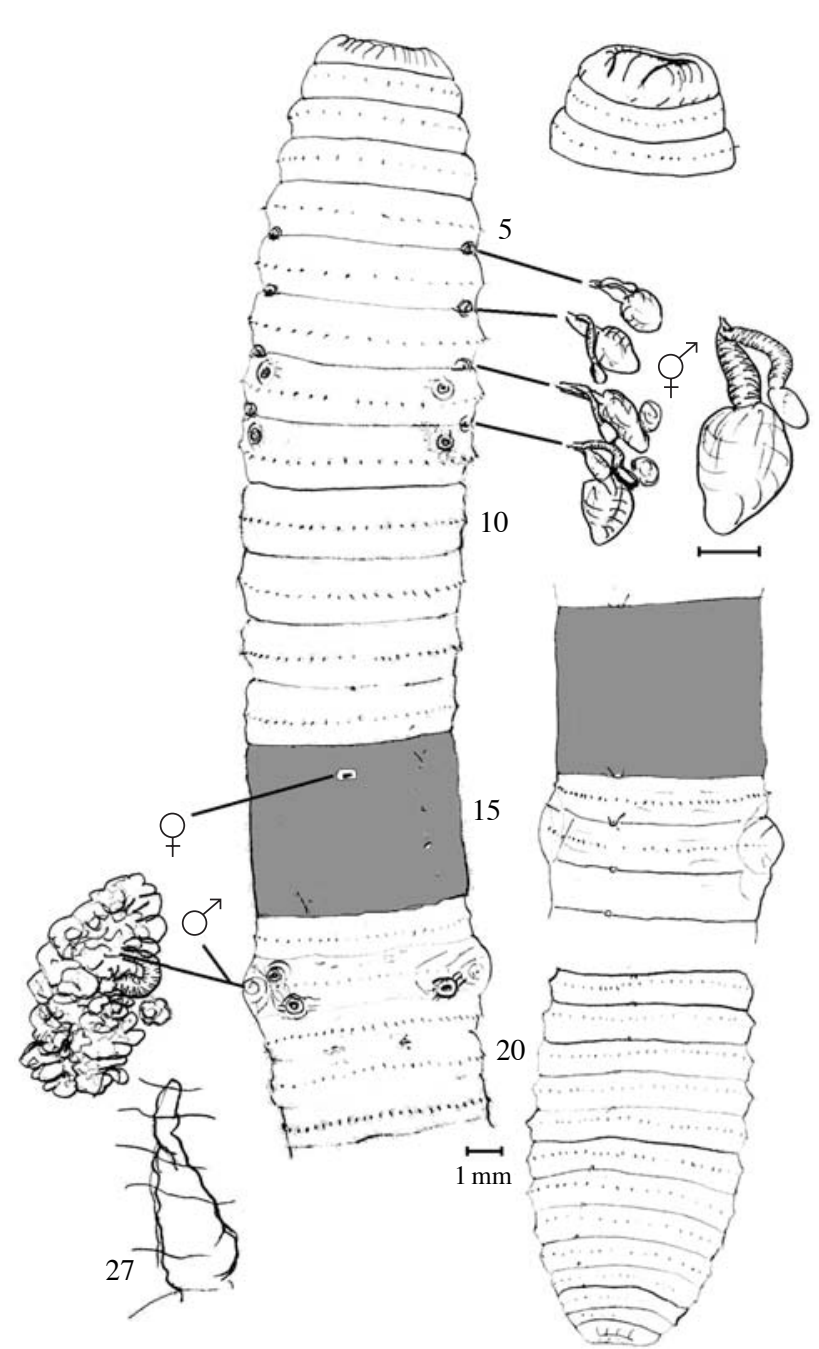

Fig. 3. A. carnosus - sketch of Hikone specimen (Tokyo An-460 DNA JET-112) showing ventral view of anterior and dorsal views of prostomium, clitellum and flared male pores posteriorly (other specimen An-461 and JET samples 113-115 agreed 100\%). This specimen resembles $P$. pingi (cf Fig. 11) but its DNA agrees with A. carnosus (as in Fig. 1).

from Seoul. DNA data is unequivocal that $A$. carnosus naribunji is intermediate between $A$. carnosus carnosus from Japan and Korea and A. carnosus roki from both Incheon and Busan (see Blakemore and Lee, 2013, fig. 1). That no similar taxon is yet reported on GenBank provides supporting justification for its consideration as unique, albeit as a mostly molecular taxon (Appendix).

Other A. carnosus carnosus proper specimens on Ulleungdo tended to be slightly smaller and paler and, significantly, either had no other GMs or had a pair of markings anterio-median to the male pores and thus mostly complied with Kobayashi's (1936) Types I \& I - the most common manifestation. Song \& Paik's (1969), "Pheretima sp. 1" from Ulleungdo - that was named as A. baemsagolensis Hong \& James, 2001 but is yet another synonym of A. carnosus - similarly has Kobayashi's (1936) Types I \& I form. In contrast, A. carnosus roki typically has Kobayashi's (1936) Types II \& I form - a combination that should now be excluded from A. carnosus carnosus (see Blakemore and Lee, 2013). For A. carnosus naribunji sub-sp. nov., the GMs comply with Kobayashi's (1936) Types I \& II markings that should similarly be considered for exclusion from A. carnosus. That is, those specimens with GMs posterio-median to male pores. It is important to note that this arrangement is particularly similar to those occurring near the male pores in A. pingi as described below and shown to be a synonym of A. carnosus carnosus proper. The issue now is defining the scope and relationships of all A. carnosus sub-species in Japan, Korea and adjacent countries as well as any transported specimens.

\section{Amynthas corticis (Kinberg, 1867)}

(Figs. 4-7)

A partial annotated synonymy from (Blakemore, 2012d) with discussion in Remarks.

Perichaeta corticis Kinberg, 1867: 102. [Type locality Oahu, the main Hawaii Island. Types in the Stockholm Museum: 1947, possibly immature].

Megascolex (Perichaeta) diffringens Baird, 1869a: 40 and 1869b: 387. [Original account of this species is presented below in full under $A$. diffringens].

?[Megascolex (Perichaeta) sanctaehelenae Baird, 1873: 96. See A. diffringens below].

Perichaeta subquadrangula Grube, 1877: 553. [Type locality Fiji. Types in Berlin, 705]. [Note: Due to its poor description, Sims \& Easton (1972: 224) had this (misdated as "1868: 36") incertae sedis, but Easton (1984: 118) placed it in synonymy of corticis].

Megascolex indicus Horst, 1883: 186 (part? Non types). [Type-locality: Sumatra and Java. Types reportedly in Leiden Museum 1917-8 - see Sims \& Easton (1972: 260)].

Perichaeta peregrina Fletcher, 1886/7: 969. [Type locality plant pots from a nursery in Sydney, believed introduced from Mauritius. Types in Sydney? Untraceable at Macleay Museum, at the University of Sydney - Dr Margaret Humphrey, Curator, Entomology pers. comm. to RJB, April, 2005]. [Authorship dated "1886" eg by Michaelsen (1900); dated "1887" by Sims \& Easton (1972: 239, 260); Easton (1984: 119). "The part containing Fletcher's paper was actually published on 17 November 1886" - M.L. Augee, Editor, Proc. Linn. Soc. NSW pers. comm. to RJB (Oct. 2004) although the journal, now pubished online, is clearly dated “1887”]; Michaelsen, 1900: 293 (syn. ?molokaiensis). Amyntas peregrinus: Beddard, 1900: 414 (syns. molokaiensis, floweri).

?Perichaeta mirabilis Bourne, 1887: 668. [Naduvatam, 
A

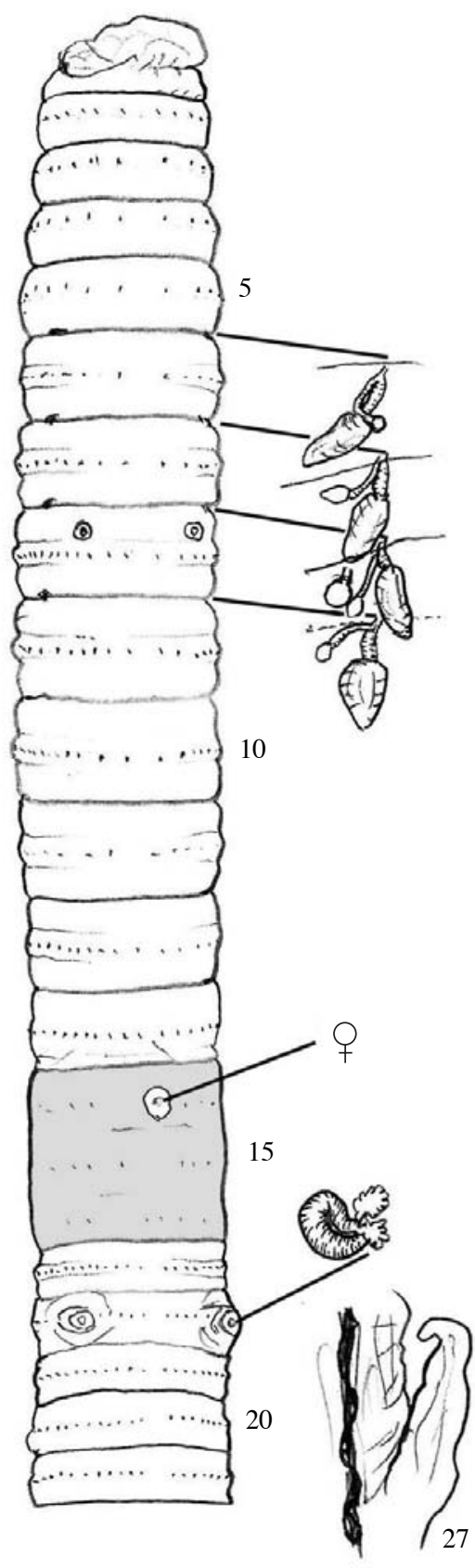

B

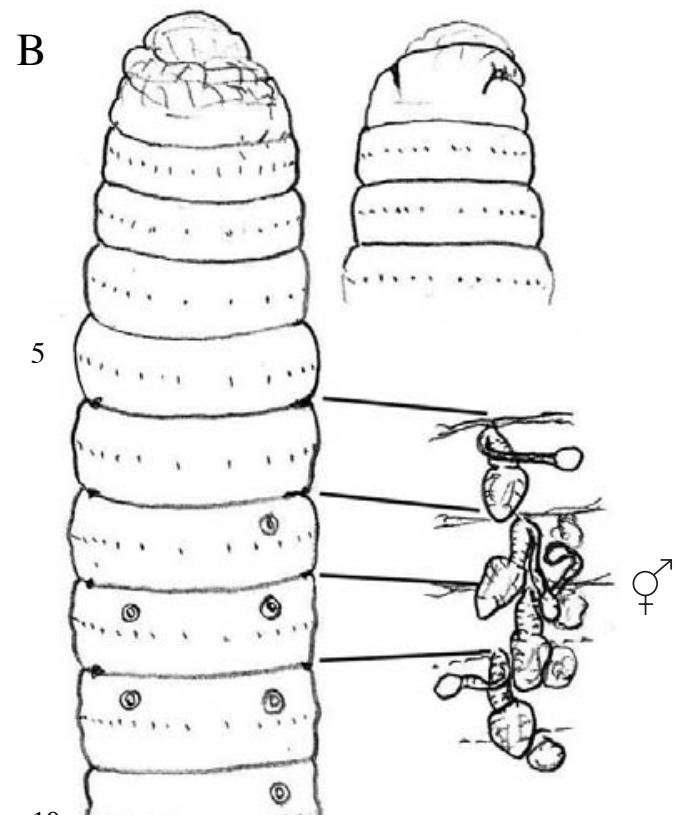

10

().

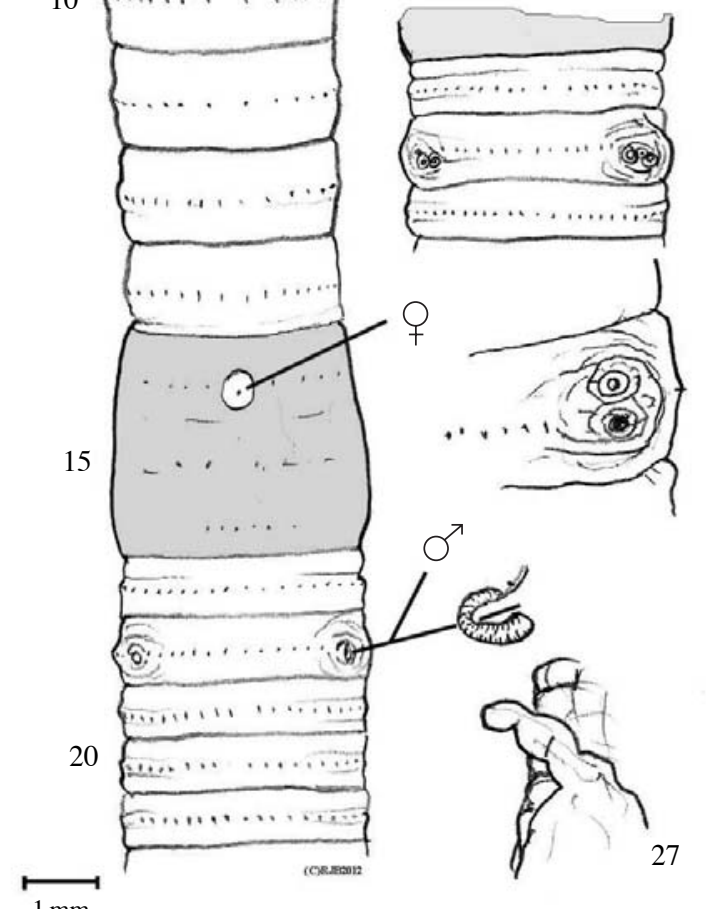

Amynthas corticis (NZ \& ROK)

Fig. 4. Amynthas corticis - specimens A from Arataki, New Zealand (providing DNA WM7 specimen in Auckland Museum) and B Korea (providing DNA WM9) plus male pores of its two sympatric specimens for comparison (specimens NIBRIV249903-4). Note: these superficially similar specimens separate genetically (see Fig. 1).

Nilgiris, India. Types?].

Perichaeta heterochaeta Michaelsen, 1891: 6. [Azores. Types lost]; Michaelsen, 1900: 275 (under indica with other synonyms ?corticis, diffringens, ungulata (laps. pro cingulata), heteropoda and nipponica).
Amyntas heterochaeta; Beddard, 1900: 416 (Hawaiian earthworms, syn. nipponica? - described with no GMs or paired or unilateral in some of 6-9).

Pheretima heterochaeta: Michaelsen, 1900; etc.

?Perichaeta ijimae Rosa, 1891: 402. [Type locality Japan. 

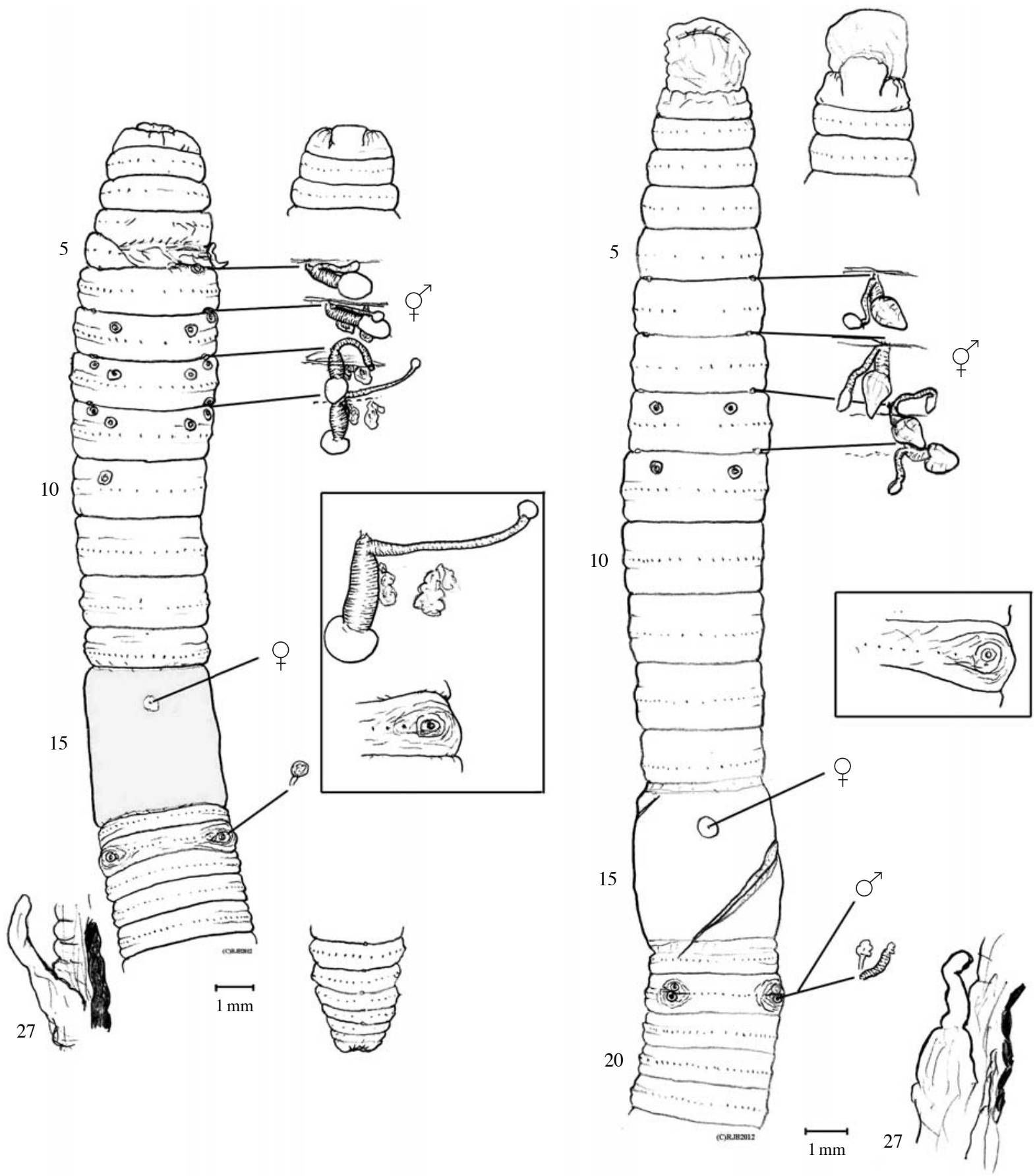

Fig. 5. Amynthas corticis - specimen from Korea (DNA sample WO4) showing X2 enlargement of aberrant spermatheca and the male pore 18rhs; second specimen (DNA sample WO5) and [boxed] the male pore 18rhs of third specimen (DNA WO6). Note: these specimens with superficially different GMs comply genetically (see Fig. 1 and cf Fig. 10).

Types Vienna?]. [Spermathecae adiverticulate in 5/6/ 7/8, cf A. gracilis, A. micronarius].

[Perichaeta divergens Michaelsen, 1892: 243, tab. 13, fig. 21. Type locality Japan. Types Berlin, 2116 "Hilgendorf rp.", now lost and not located in BMNH either - RJB pers. obs.. Prostates aborted, seems separable on its serrate intestinal caeca].

[Pheretima divergens: Michaelsen, 1900: 264, 314317 (?syn. fuscata, campestris, kamakurensis, parvula, heteropoda, obscura, scholastica, decimpapillata, flavescens, producta, micronaria - all Goto \& Hatai, 1898; all from Japan; but the latter one, at least, is 


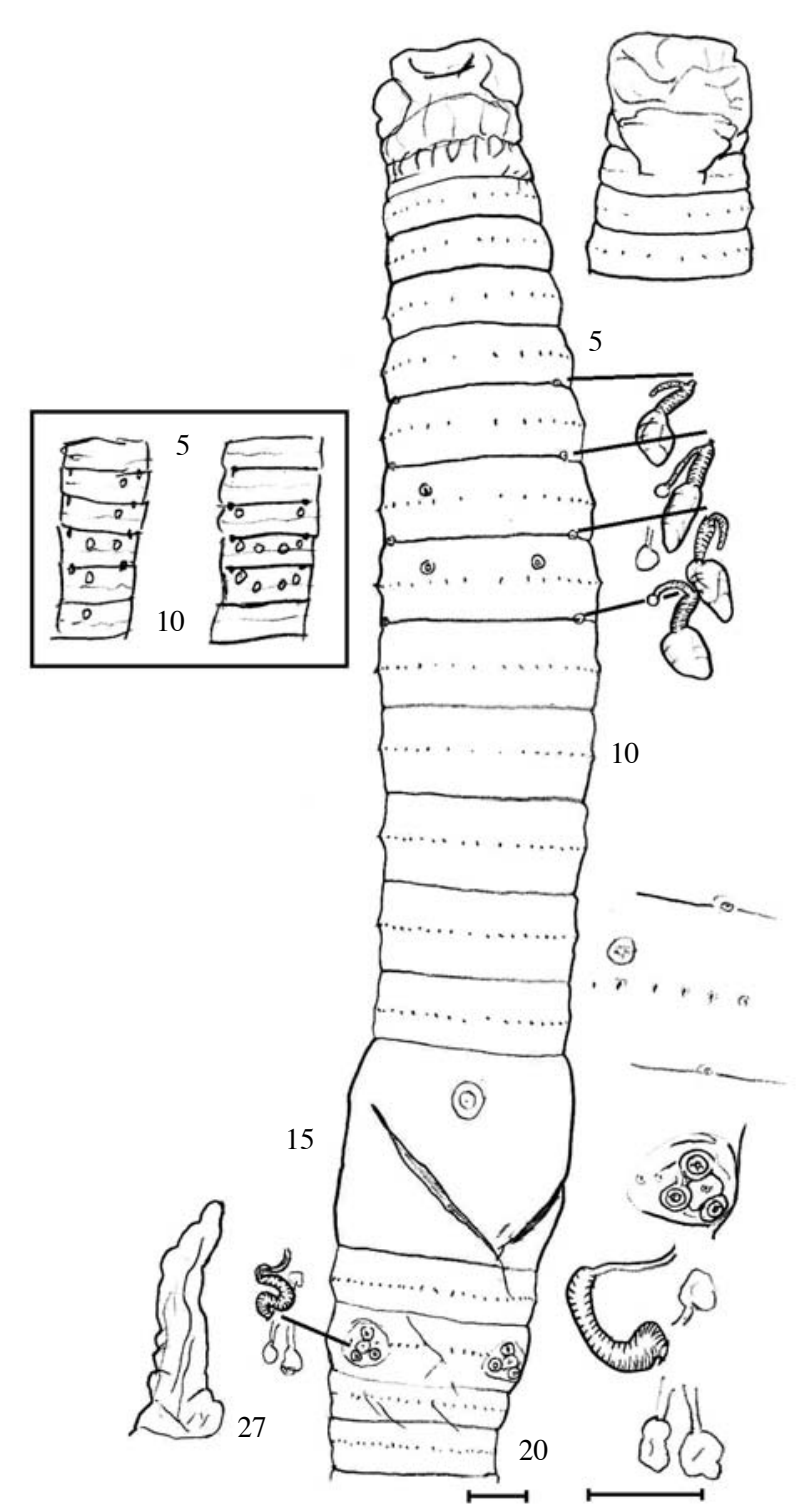

Fig. 6. Amynthas corticis - specimen from Incheon (NIBRIV261267, DNA sample w59) with enlargements of main characteristics; [boxed are sketches of GMs of two other sympatric specimens IV261270]. The unusual arrangement of GMs around male pores suggested a new taxon but DNA did not differ from other A. corticis specimens (Fig. 1; Appendix and cf. A. hongkongensis, A. hatomajimensis and A. cruxus).

doubtful); Ohfuchi, 1937: 67-108, tabs. 1-25, figs. 1128 (without including any synonyms of Michaelsen!)].

Perichaeta nipponica Beddard, 1892: 760. [Japan. Type BMNH 1904:10:5:993 - no longer traceable in the collection there, E. Sherlock per. comm., RJB pers. obs. VI.2013].

Pheretima nipponica: Michaelsen, 1900; Ohfuchi, 1937: 108-133, fig. 29-36.

?Perichaeta perkinsi Beddard, 1896: 198. [From Hawaii. Types missing? Not found in BMNH pers. obs. RJB
VI.2013]. [Spermathecae 5/6/7/8/9; possibly a separate, although exotic, species/variety].

?Perichaeta molokaiensis Beddard, 1896: 201. [From Hawaii. Types missing? Not found in BMNH pers. obs. RJB VI.2013]. [Spermathecae 5/6/7/8/9].

Perichaeta heteropoda Goto \& Hatai, 1898: 69. [Japan. Types none - confirmed RJB (Blakemore \& Ueshima (2011)]. [Spermathecae 5/6/7/8/9. GM in 6-9 paired and pre-setal - only markings in 6 separate it from $A$. diffringens types, pers. obs. Name yet retained by some contemporary Japanese and/or Koreans sometimes as "Amynthas heteropodus" or "A. heteropoda", or misspelt as "heteropadus"; for example Hong \& Kim (2009) and Dr Young Hong again presented this as a contemporary Korean species at a seminar in Incheon in September, 2012].

Pheretima heteropoda: Ohfuchi, 1937: 42-50, figs. 47 (part? cf A. diffingens here).

Pheretima marenzelleri Cognetti, 1906: 780, figs. 5-6. [From Yokohama. Type Vienna? Kobayashi, 1938: 407, figs. 1a-b (recorded from Hokkaido and, p. 408 footnote: "non Ohfuchi, 1936"]); redescribed by Blakemore (2012c); yet quoted as valid by Shen et al. (2013) for some reason].

?Pheretima silvestrii Cognetti, 1909: 266. [From Hawaii. Types Turin 194].

Pheretima divergens yunnanensis Stephenson, 1912: 274. [From Yunnan. Type Calcutta (5062)]. [Mistaken by some subsequent workers as " $P$. browni yunnanensis". Gates (1939: 430) inspected the fragmented type(s) described below with A. diffringens].

[?Pheretima pingi Stephenson, 1925: 891 (cf A. carnosus) description below].

?Pheretima indica var. cameroni Stephenson, 1932: 47, fig. 4. [From Cameron Highlands, Malaya. Type in London, possibly missing? Not found in BMNH pers. obs. RJB VI.2013]. [A single poorly preserved specimen with spermathecae in 5/6-8/9 no nephridia on ducts; no markings - probable syn. nov. of corticis].

?Pheretima sheni Chen, 1935: 38. [From Hong Kong, eight specimens three of them aclitellate and all lacking spermathecae. Type described in the Museum of Fan Institute of Biology, Peiping (Beijing) but also claimed in US National Museum]. [Cf Amynthas robustus, A. illotus].

?Pheretima directa Chen, 1935: 47. [From Hong Kong; three specimens, the type described in the Museum of Fan Institute of Biology, Peiping but also claimed in US National Museum: 20183]. [Because some genital markings are lateral to the male pores it is possible that this is a valid species but of A. hatomajimensis as described below].

?Pheretima oyamai Ohfuchi, 1937: fig. 10, Pl. I, 3. [From Morioka, Iwate-ken and Odate, Akita-ken. Types? The 

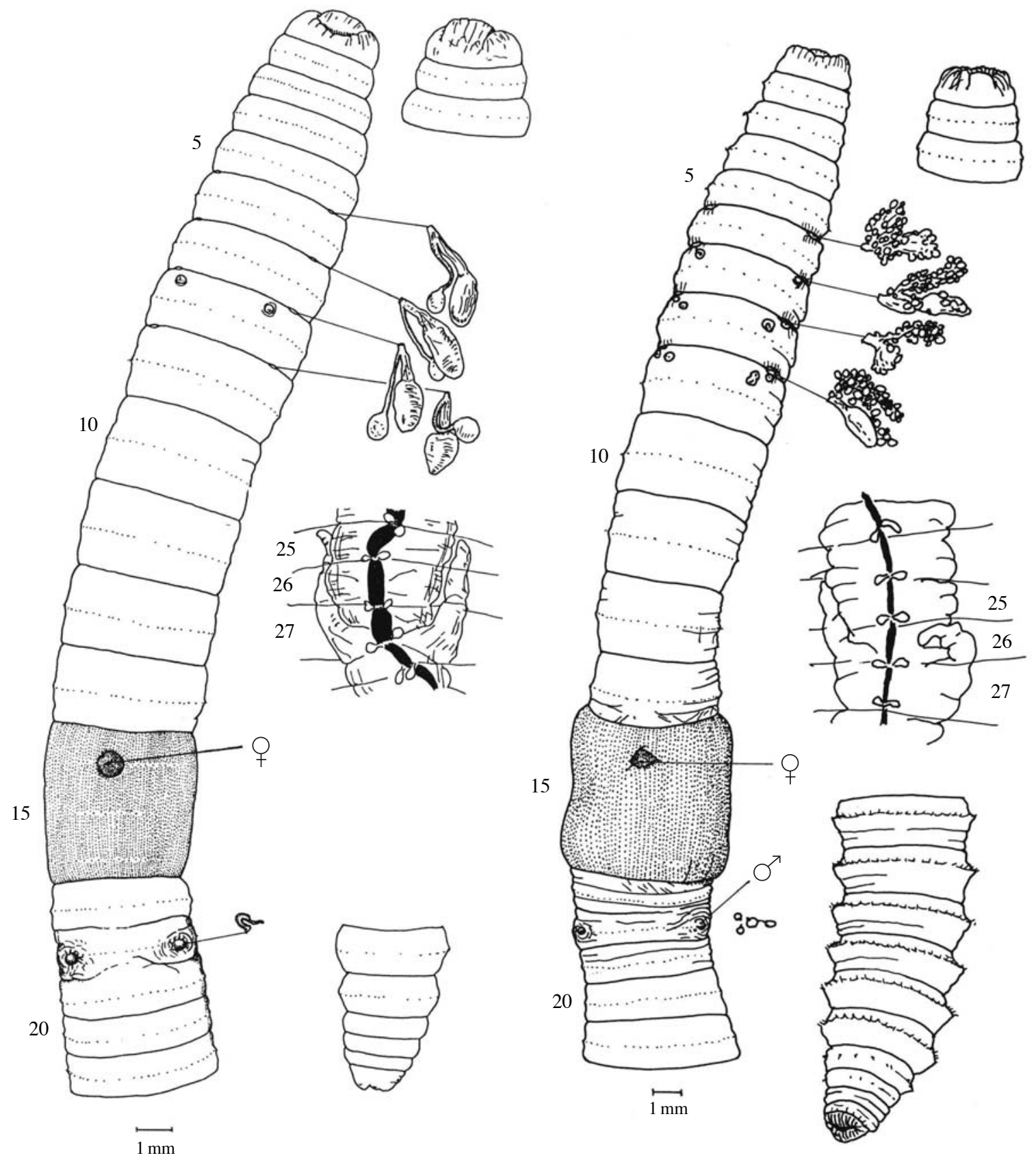

Fig. 7. Amynthas corticis - author's 1990 specimens from Queensland, Australia showing (lhs) complete and (rhs) Monocystis parasitized spermathecae (after Blakemore, 2012d).

spermathecae are adiverticulate and GMs only on 18 , exactly as he shows for his subsequent redescription of Ph. divergens but caeca are simple and smooth. Cf A. carnosus as an adiverticulate morph?].

Pheretima tajiroensis Ohfuchi, 1938: 46, figs. 12-14. [From Tajiro Island, Miyagi-ken. Types not known. Parasitized spermathecae \& prostates mistaken as characteristics].

?Pheretima homoseta Chen, 1938: 414. [From Hainan.
Types? Restored in some latest accounts as Amynthas homosetus but without good reason. Originally as Pheretima homochaeta, an 'incorrect original spelling' (under ICZN, 1999: Art. 32.5.1.1) subsequently corrected to P. homoseta by Chen (1938) in an ERRATA sheet].

?Pheretima morii Kobayashi, 1938: 161, fig. 15. [From Korea. Types unknown. Spermathecae 5/6/7/8/9, GMs in 7-9, and just before male pores; prostates aborted]. 
?Pheretima toriii Ohfuchi, 1941: 244, fig. 2. [From cave, Oita-ken. Types? Small $40 \mathrm{~mm}$ and lacking GMs].

?Pheretima clerica Benham 1947: 423, Pl. 1, figs. 1-5. [From Gisborne district in NZ. Types? There appears some slight difference in that male pores are in small slits and there is a genital papilla on the inside of each; prostates are present].

Pheretima campestris Lee, 1952: 39. [From New Zealand (and Kermadec). Types Te Papa, W.000494]. [Placed in synonymy of $P$. peregrina (Fletcher, 1887) (=Amynthas corticis) non P. campestris Goto \& Hatai, 1898 (?=Amynthas robustus)]. [Non senior secondary synonym P. campestris Goto \& Hatai, 1898: 67].

?Pheretima hatomajimensis Ohfuchi, 1957: 245 [From Japan. Types? - described below].

Amynthas corticus (sic lapsus pro corticis): Sims \& Easton, 1972: 235, 241; Easton, 1981: 49-50 (syn. diffringens, divergens, ?hatomajimensis, heterochaeta, heteropoda, ?ijimae, indica Horst, 1883: 186 (sic lapsus Easton, 1981 pro indicus), ?marenzelleri, nipponica, oyamai, tajiroensis, toriii).

Amynthas corticis: Easton 1982: 726-728 (syn. diffringens, peregrina, heterochaeta); Easton, 1984: 118 (syn. subquadrangula); Sims \& Gerard, 1985 (syn. diffringens, indicus, nipponica); Blakemore, 2012d (listing above syns. plus about a dozen extra synonyms from Japan).

Taxonomic Notes. The name corticis is sometimes misspelt "corticus" or "coritcis". It is not certain that all subspecis of Megascolex indicus Horst, 1883 s. strict. (cf Pheretima darnleiensis (Fletcher, 1886)) follow it into synonymy of corticis; these subspecies are listed by Sims \& Easton (1972: 235) as: Amynthas indicus cameroni (Stephenson, 1932) from the Malay Peninsula; Amynthas indicus ceylonicus (Michaelsen, 1897: 246) from Sri Lanka; and Amynthas perkinsi (Beddard, 1896) from Halemanua and Kauai, Hawaii which was included by Michaelsen (1900: 276) as a 'variety' of indica. It appears to me that there is nothing substantial in the description of A. cameroni to differentiate it from A. corticis; and certainly the latter two taxa are not endemic to their localities. Stephenson (1923: 302) includes ceylonica (and its variant mispelling "ceylanensis" in $P$. heterochaeta (=A. corticis); and Gates (1972: 149) includes A. perkinsi as a possible synonym of corticis in a footnote where he lists the names under which specimens of $P$. diffringens have been referred to as, or have been types of $A$. perkinsi was based on two specimens, neither with genital markings, that, however, had complete spermathecae and prostates; probably the same as A. corticis as is the almost identical A. molokaiensis (Beddard, 1986).

Diagnosis. Amynthas with four pairs of spermathecal pores ca. 0.3 body circumference apart in furrows $5 / 6 / 7$ / $8 / 9$ [all, or just posterior pair (?or anterior pair) rarely lacking]; genital markings small paired and variable near spermathecal and male pores, often widely paired. Intestinal caeca simple with smooth margins (or incised with age in older specimens $\mathrm{cf} A$. divergens?) originating near segment 27. Prostates often aborted, sometimes present. Parthenogenetic and parasitized morphs common (eg lacking prostate glands and/or with partially or wholly aborted or adiverticulate spermathecae).

Distribution. According to Gates (1972) its synonym occurrence is "everywhere, including Europe". It is the most widely distributed of the allochthonous species of the pheretimoid group, having been recorded from temperate and tropical regions throughout the world.

Remarks. Amynthas corticis (Kinberg, 1867) type-locality was in Oahu and it was also from Hawaii that Beddard (1900: 415) claimed "Amyntas peregrinus Fletcher" [=A. peregrinus (Fletcher, 1896/7) originally from Sydney] and placed in synonymy his own $P$. molokaiensis Beddard, 1896 that seems indistinguishable from his subsequent description (Beddard, 1900: 416) of "Amyntas heterochaeta Mich.” (sic) [=Amynthas heterochaetus (Michaelsen, 1891)] from there. These four taxa are identified as having spermathecal pores in 5/6/7/8/9 and either having or lacking posterior GMs and prostate glands and have since been placed under A. corticis. Their superficial male pores and lack of copulatory sacs qualifies them for Amynthas and, moreover, they thus comply within the range of characters allowed for in Ohfuchi's (1937: 42) detailed account of Pheretima heteropoda (Goto and Hatai, 1899) that should also be considered at least a partial synonym due to his "variations" with many arrangements, including total absence, of anterior GMs (Ohfuchi, 1937: text-figs. 5, 6). This situation may be compared to Blakemore (2013a: fig. 2) where anterior markings in an A. corticis are present or unilaterally absent making use of this distinction for taxa separation irrelevant.

Ohfuchi (1937: 108) was inconsistent when he described prior P. nipponica (Beddard, 1893) that seems indistinguishable from his A. heteropoda (Goto \& Hatai, 1899) since he claimed both lacked markings around male pores - the only difference was in length of spermathecal diverticula, but these he described as highly variable in $P$. nipponica. Thus, Ohfuchi's concept of $P$. heteropoda should have been subsumed under $P$. nipponica. All these taxa are now included in $A$. corticis as indicated by Easton (1981) and remain so unless proven otherwise, except perhaps for P. divergens (Michaelsen, 1892) with its serrate caeca.

Michaelsen (1900: 264, 314-317) redescribed his Pheretima divergens with possible synonyms $P$. fuscata, campestris, kamakurensis, parvula, heteropoda, obscura, scholastica, decimpapillata, flavescens, producta, and micronaria - all by Goto \& Hatai (1898) and all from Japan (and all ignored by Ohfuchi, 1937!); and all but 
the latter one, at least, doubtfully separate, even to this day. Ohfuchi (1937: 100, text-fig. 23, 3-5) differentiated his specimens of Pheretima divergens (Michaelsen, 1892) from other taxa due to their serrate (or at least deeply indented) intestinal caecal ventra. On this latter basis alone the taxon Amynthas divergens is apparently separable from both $A$. corticis and A. diffringens, as well as from A. carnosus (Goto \& Hatai, 1899) which Ohfuchi (1937: 56; text-fig. 9) also describes in some detail (he retained it because he found it to have markings around the male pores, as in his A. divergens, but to lack the serrate caeca). Gates (1972) kept $P$. divergens in synonymy of $P$. diffringens, although Ohfuchi's (1937: 67-108) detailed redescription showed divergens to have great variation in GMs and wide distribution in Japan. It is likely this taxon, herein restored, also occurs in Korea (and elsewhere?) either as a native or an introduction.

A more complete descriptions of A. corticis, its synonymy, distribution and bibliography are found in Blakemore (2012d; 2013a; 2013b) where some DNA data are also presented (but unfortunately not from historical primary types).

Korean specimens providing DNA samples WM9, WO4, WO5, WO6 and WO56 (from Jeju) all had mega BLAST agreement of $100 \%$ along with other A. corticis specimens from Biwa (JET-120-121) and Mishima (JET 138-139) in Japan. These differed substantially from other specimens identified as "A. corticis" or " $A$. cf. heteropodus" that provided DNA samples w59 (from Incheon), WM7 from NZ plus other specimens from Biwa (JET-122-123) and Mishima (JET 140-141) in Japan (in Appendix but without repeats). There seems to be at least two genetically distinct $A$. corticis lineages sharing the same name (Fig. 1). Further work is required to resolve this issue of identity of the two groups under the taxonomic Principal of Priority.

Samples providing DNA codes JET153-154 from NSW Australia that I tentatively labeled as A. cf. corticis differ substantially and are most likely closer related to A. gracilis (Kinberg, 1867); it is a small concern that the single specimen (Tokyo An-477) provided the two samples that gave slightly different results from PCR analysis (Fig. 1; Appendix).

\section{Amynthas diffringens (Baird, 1869)}

(Figs. 8-10)

Megascolex (Perichaeta) diffringens Baird, 1869a: 40, fig. 1. [Type locality and habitat: a hothouse nursery at Plas Machynlleth (house), North Wales. Types in British Museum: BMNH 1869:1:2:1 inspected as described below].

Perichaeta diffringens: Vaillant, 1872: 322. [Described with caeca present, prostates absent, four pairs of spermathecae but he miscounted segments (5/6/7/8/9?) as

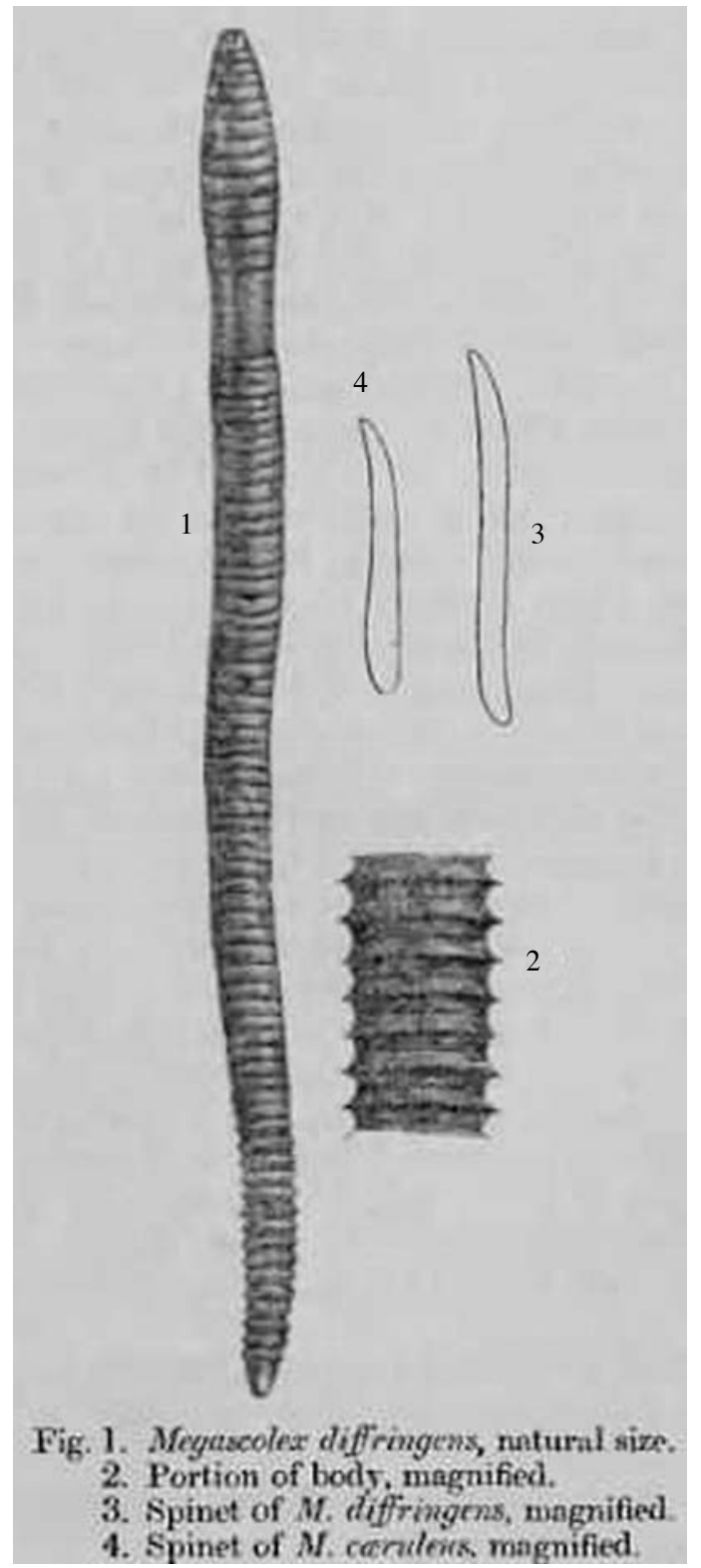

Fig. 8. Amynthas diffringens copy of Baird's original fig. 1 (habitus and a seta).

“3/4/5/6/7”].

Pheretima diffringens: Kobayashi, 1938: 158; Gates, 1939: 430 (syns. divergens var. yunnanensis, divergens (part. inc. Japanese types?), heterochaeta, mirabilis); Gates, 1943: 102 [with genital markings on 7-9 midway between furrows and setal arcs, hearts of 10 lacking, prostate glands absent but gential marking glands in 18 just median to prostatic ducts cf description below]; Chen, 1959: 9, text-fig.; Gates, 1972: 149, footnote 2: [?syns. (some in part) californica, campestris, cingulata, clerica, corticis, divergens, heterochaeta, heteropoda, indica, mirabilis, molokaiensis, nipponica, peregrina, 


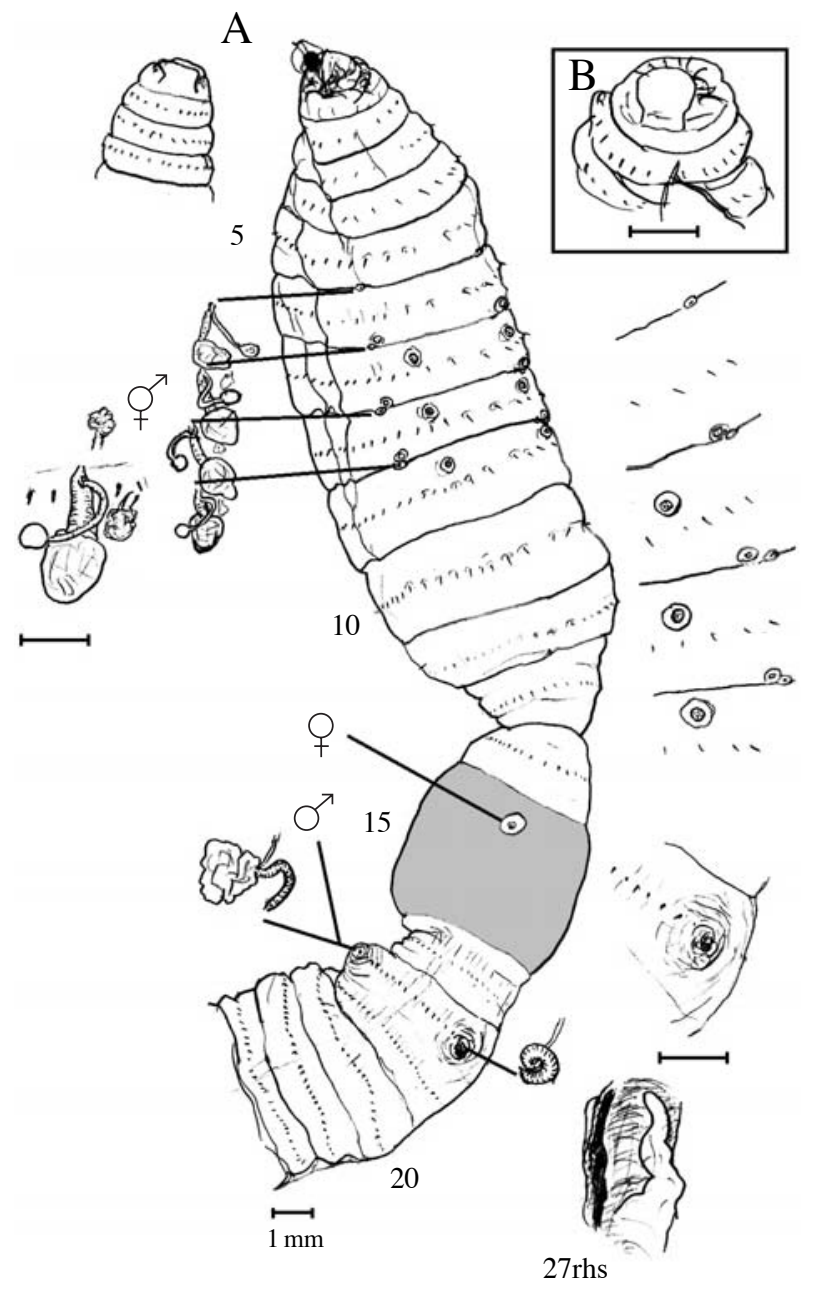

Fig. 9. Amynthas diffringens - A the previously dissected syntype showing prostomium, ventral habitus with spermathecae and prostates in situ and caecum 27rhs; X2 enlargements are of a 9lhs spermathea, 5/6/7/8/9rhs spemathecal pores and 18rhs male pore; B A. sanctaehelenae prostomium of previously dissected subadult type.

perkinsi, sanctae-helenae, silvestrii, tajiroensis, torii, ?carnosa, ?directa, ?homoseta, ?kyamikia, ?morii, ?oyamai, ?pingi]; Gates, 1972: 177 (syn. heterochaeta, divergens yunnanensis, mirabilis); Gates, 1972: 217 (?syn sheni); Gates, 1972: 18, 27 (syn. divergens, heterochaeta, heteropoda, indica, nipponica, oyamai, tajiroensis, ?toriii); 1982: 44.

Amynthas diffringens: Easton, 1979: 119 (syn. ?subquadrangula); Hong \& James, 2001: 92 (i.e. more than 20 years after it was shown to be a synonym of $A$. corticis!).

Megascolex (Perichaeta) Sanctae-Helenae Baird, 1873: 96. [From high ground at St Helena, I. or J.C. Melliss, Esq. Type in British Museum: BMNH 1869:6:16:5 inspected by RJB 24.IV.2012 and 17.VI.2013 found to be juvenile, $40 \mathrm{~mm}$ long with 104 segments, damaged by dissection with spermathecae and prostates absent or removed, status: incertae sedis]. Baird gives the impression that more than one specimen was found, only one now remains. His description in full:

"Body consisting of about 86 rings, which are more distinct at the two extremities than in the centre. The 11 or 12 rings at each end, have an acute ridge or keel in the centre; those of the middle portion of the body have the keel flattened. The body of the rings is finely striated. Setae short, of a dark colour at the posterior extremity, rather distant from each other. In the centre of the body and at the anterior extremity they appear (in the specimen from which this description was drawn up) retracted, leaving only a mark where they are situated. The first 7 or 8 rings, at the anterior extremity, are strongly rugose or wrinkled. Length from 1 inch and 9 lines to 3 inches. Hab. High ground at St. Helena, J.C. Melliss, Esq."]. [Type locality St Helena. Type in British Museum: BMNH 1869: 6:16:5. Held incertae sedis by Michaelsen (1900: 518) with which I now concur].

Pheretima divergens var. yunnanensis Stephenson, 1912: 274. [Type locality: Tengyueh, Yunnan; type in Indian Museum according to Gates (1939: 430-431) and "quite clearly" a synonym of $P$. diffringens hence its inclusion here, now questionable].

Note. Gates (1939: 340) also inspected Pheretima barbadensis BMNH 1904:10.5.1219.1228 from Hong Kong that he later (1939: 446, 454) said also contained specimens of $P$. hawayana and P. morrisi; Pheretima morrisi 1904:10.5.453 also from Hong Kong that he also later listed (1939: 446, 454) as containing P. hawayana!; from US National Museum Pheretima corrugata Chen, 1931 (part. - a paratype from Szechuan inspected by Gates, 1939: 340, not holotype). Therefore the name is debatably a junior synonym, although the paratype was "rather obviously to be referred to $P$. diffringens".

Material examined. Types in Natural History Museum, London: BMNH 1869:1:2:1, inspected by RJB 24.IV.2012 and 17-20.VI.2013 compared to Baird (1869a) "Description of a new species of earth-worm (Megascolex diffringens) found in North Wales". Syntypes labeled: "1869. 1.2.1 TYPE Plas Machynlleth Montgomeryshire, N. Wales". The jar (not original) contained nine mature specimens (four previously dissected dorsally), one juvenile and three portions; all were in good condition, flexible but rather macerated. The largest specimen was figured and is redescribed in detail here. Baird (1869b: 387) in "Additonal remarks on the Megascolex diffringens" had specimens sent from Lady Cullum's hothouse at Hardwick House near Bury St. Edmunds, Suffolk where the gardener says it resided for about 14 years although for 20 years he knew it from Glevering Hall near Woodbridge, Suffolk - these latter two localities unconfirmed 


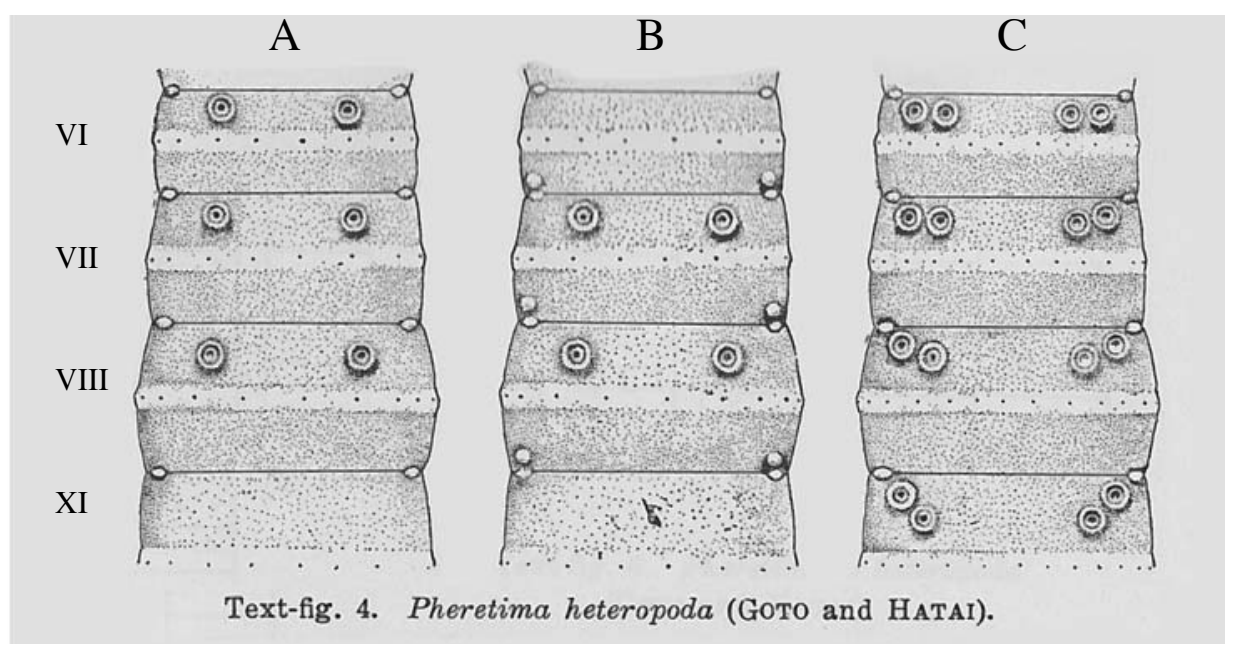

Fig. 10. A. corticis - Ohfuchi's (1937: fig. 4) three representative forms of his ' $P$. heteropoda' from Japan that is now a synonym of A. corticis. However, the central figure agrees almost exactly with $A$. diffringens types (as described herein) having markings just above spermathecal pores (Fig. 9) while the outer two specimens seem to agree with those A. corticis Korean specimens figured that provided DNA sample WO4WO5 (Figs. 1, 5).

and material not located.

Also Megascolex sanctae-helenae (sic) Baird "1869.6. 16.5 TYPE Island of St Helena I.C. Melliss Esq." a single brittle subadult (inspected).

Distribution. From hothouses in Wales and possibly England from original descriptions, and from Japan and probably Korea from subsequent descriptions of a (partial?) synonym A. heteropodus (Goto \& Hatai, 1899).

Original description (in full). Length 4-5 inches (100$125 \mathrm{~mm}$ ) and "3 lines in circumference" [secondary annulations], with 104-105 segments. Lives in compost, nocturnal wanderer, very active when disturbed and readily breaks into fragments (hence its name). Clitellum from 14. Setae about 60 per segment. Darkly pigmented dorsum and pale ventrum.

Redescription of Syntypes. Longest complete specimen $120 \mathrm{~mm}$ (others smaller). Prostomium open epilobous. First dorsal pore in 11/12 (a non-funtional dot in 10/11). Setae fewer in anterior ventrum but more numerous posteriorly, numbering approximately 40 on 10 and ca. 56 on 20. Spermathecal pores in 5/6/7/8/9. Often with smaller ancilliary markings in 6-8 just prior (anterio-median) to spermathecal pores as seen in several specimens. Other GMs mostly widely paired papillae, presetal in 7-9 (in five of nine specimens), in $7 \& 8$ in two (one with other markings behind the male pores, the other without these), just in 8 in another, and indeterminate in the final specimen. Some variability manifest, with some markings missing. Male pores superficial with approximately 14 setae intervening (GMs present behind male pores as noted in one specimen).

Internally septa to $7 / 8$ are thin, $8 / 9$ is thick but incomplete to base of gizzard, or absent in some specimens;
$8 / 9$ is aborted. Spermathecae as figured in 6-9. Glands associated with GMs. Holandric with seminal vesicles in $11 \& 12$. Ovaries in 13; ovisacs not seen. Last hearts in 13. Intestine commences in 14 in some, but wider in 15 or 16 in other specimens; intestinal caeca are simple from 27. Prostates with racemose glands on $18 \mathrm{hs}$ in two specimens but mostly the glands absent and only duct present.

Remarks. Amynthas diffringens (Baird, 1869) was first put under A. corticis (Kinberg, 1876) (misspelt as "corticus") by Easton (1981: 49) for which he has synonymy of: "diffringens Baird, 1869: 40; (syn. divergens, heterochaeta, heteropoda, indica, nipponica, oyamai, tajaroensis [sic, lapsus tajiroensis], ?torii) Gates, 1972c: 18"; several other synonyms now probable (see Blakemore, 2012d). Also claimed as "syn. nov." of corticis by Easton (1982: 726) and by Sims \& Gerard (1985). Amynthas corticis is a widely distributed cosmopolitan species-complex (eg by Blakemore, 2003: 14, 2012d) having accrued a large number of synonyms. The problem is that the extant type of A. corticis from Hawaii is an immature form. Thus the definition is somewhat vague and, in its broadest definitions at least, it encompassed earlier A. diffringens definitions. Here the redescription of the syntypes allows a more precise characterization of $A$. diffringens and its resurrection is proposed based mainly on its greater number of genital markings anteriorly, especially those in front of spermathecal pores (and fewer, or none, near the male pores) compared to what is most often found in a A. corticis that is seemingly now restricted in its definition to exclude these markings anteriorly of spermathecal pores (although they may occur posteriorly to spermathecal pores as in specimen NIBRIV249906 pro- 
viding DNA WO4).

It should be noted that $A$. corticis often has markings near the male pores and that the male pores may be entirely missing with only the marking remaining. Related to this is the usual absence of the glandular portion of the prostate, if not deletion of the entire organ. Specimens that are degraded or immature require DNA to determine their identity.

Since no specimens were retrieved from the UK typelocality, the task now is to find live specimens from the unknown original Asian homeland agreeing with $A$. diffringens and to extract DNA to unambiguously define boundaries of this taxon. Those from Fukuura, Japan described by Ohfuchi (1937: 45) are perhaps closest thus far known.

Ohfuchi (1937) did not consider and was probably unaware of $P$. diffringens when he produced his detailed accounts - although his associate Kobayashi (1938) knew of diffringens and included Ohfuchi's nipponica in synonymy (see its synonymy above). However, Ofushi's description of P. heteropoda (Goto \& Hatai, 1899) seems to overlap the current redescription of the types since he showed (Ohfuchi, 1937: text-fig. 4B; 5A'-F') GMs preceding some or all the spermathecal pores as well as the presence or absence of prostate glands (Ohfuchi, 1937: text-fig 7A). Thus his concept of Amynthas heteropodus should go partly under A. corticis and partly under A. diffringens as currently defined herein. The present description of A. diffringens also compares to those of other earlier workers, especially Kobayashi (1938: 158), Gates (1972, 1982) and Chen (1959: 9) that similarly overlap somewhat what has since been accepted as A. corticis s. lato.

\section{Amynthas hatomajimensis (Ohfuchi, 1957) \\ Pheretima hatomajimensis Ohfuchi, 1957: 245, fig. 20. [From Ryukus, Japan. Types?].}

Diagnosis. Length $73-94 \mathrm{~mm}$ by 3-4.5 $\mathrm{mm}$ with $75-93$ segments. Spermathecal pores in 5/6/7/8/9. GM in a cross-shape internal to, above and below male pores on 18 , plus another pair anteriomedian and postsetally on 18. Intestinal caeca simple in 26.

Distribution. Hatoma-jima island, Ryukus. Collected $1^{\text {st }}$ April, 1936.

Remarks. Said to resemble A. pingi its lack of spermathecal papillae and cross-shaped GMs indicate its propable separation from both A. corticis/diffringens and A. carnosus/pingi although combined as a possible synonym of A. corticis by Easton (1981: 49-50).

Now separated is Metaphire yamadai (Hatai, 1930) that has spermathecae in 6/7/8/9 and possibly includes A. pectiniferus (Michaelsen, 1931) both of which Gates $(1935 ; 1939)$ implicated in P. pingi as discussed in Blake- more (2012a). However, similar taxa now are A. hongkongensis (Michaelsen, 1910) from China and A. cruxus Tsai \& Shen in Tsai, Shen, Tsai and Lee, 2007: 357 from Taiwan that seem to belong to the A. corticis group and similar to current Incheon specimens of $A$. corticis and so these taxa should be reevaluated.

\section{Amynthas pingi (Stephenson, 1925) [=A. carnosus $]$}

(Fig. 11)

Pheretima pingi Stephenson, 1925: 891, text fig. 1 (of testis sac), plate II fig. 7 (of a likely parasitised spermatheca). [Type locality Nanking, China. Syntype in London, BMNH 1924:11:29:5]; Chen, 1933: 228, fig. 15; (non Gates, 1935: 14); 1936: 298 (syn. fornicata); Gates, 1939: 465 (syn. kyamikia); ?Ishizuka, 2001: 82, fig. 46 (mislabeled "Pheretima pingi Chen, 1936") exactly the same as $P$. carnosa in fig. 9 of Chen (1959) so probably is the same.

?[P. hongkongensis Michaelsen, 1910: 107. Type locality Hongkong, type in Hamburg, V9084 (inspected by Gates, 1939: 446)].

?[Pheretima fornicata Gates, 1935: 9. [From "Tatsienlu, Szechwan" now called Kangding or Dardo (from the Tibetan name Darzedo) in the Garze Tibetan Autonomous Prefecture in western Sichuan. Types in US Natl. Mus. no. 20099]; Chen, 1936: 296 (examined type); Gates, 1939: 434. [It lacks genital markings but is otherwise stated to be similar to $P$. hongkongensis that has only a pair of small marking immediately median to male pores on 18 - see fornicata description below].

Amynthas pingi: Sims \& Easton, 1972: 235 (diffringensgroup); Blakemore, 2012d; 2013a; 2013b.

Material examined. London type a single previously dissected mature specimen, labeled: "Pheretima ping $i$ 1924.11.29.5 HOLOTYPE (sic) Nanking, China Don. Prof C. Ping"; the sample jar contained a smaller vial containing a spermatheca (8rhs) with a note "Spermatheca from new Chinese worm (Prof. Ping)" in Stephenson's hand.

Diagnosis. Amynthas with spermathecal pores in 5/6/7/ 8/9 [as in A. corticis and A. carnosus cf A. pingi chungkingensis (Chen, 1936) with spermathecal pores in 6/7/8/9 now separated at species level]. Genital markings paired ventrally on 8,9 and 18 (sometimes in 19 too) (as also in A. carnosus). Intestinal caeca simple from 27 (as in $A$. carnosus $\mathrm{cf}$ A. divergens).

Distribution. Nanking, plus one (dubious?) specimen from Kuatun, Fukien at 3000-5000ft (Gates, 1943) and another dubious report from Japan (see Blakemore, 2003). Abundant in Nanking constituting $30 \%$ of worms there [Stephenson (1931: 56) on information from Dr Chen] where it was found with Metaphire houlleti. Occurrence elsewhere now suspect. 


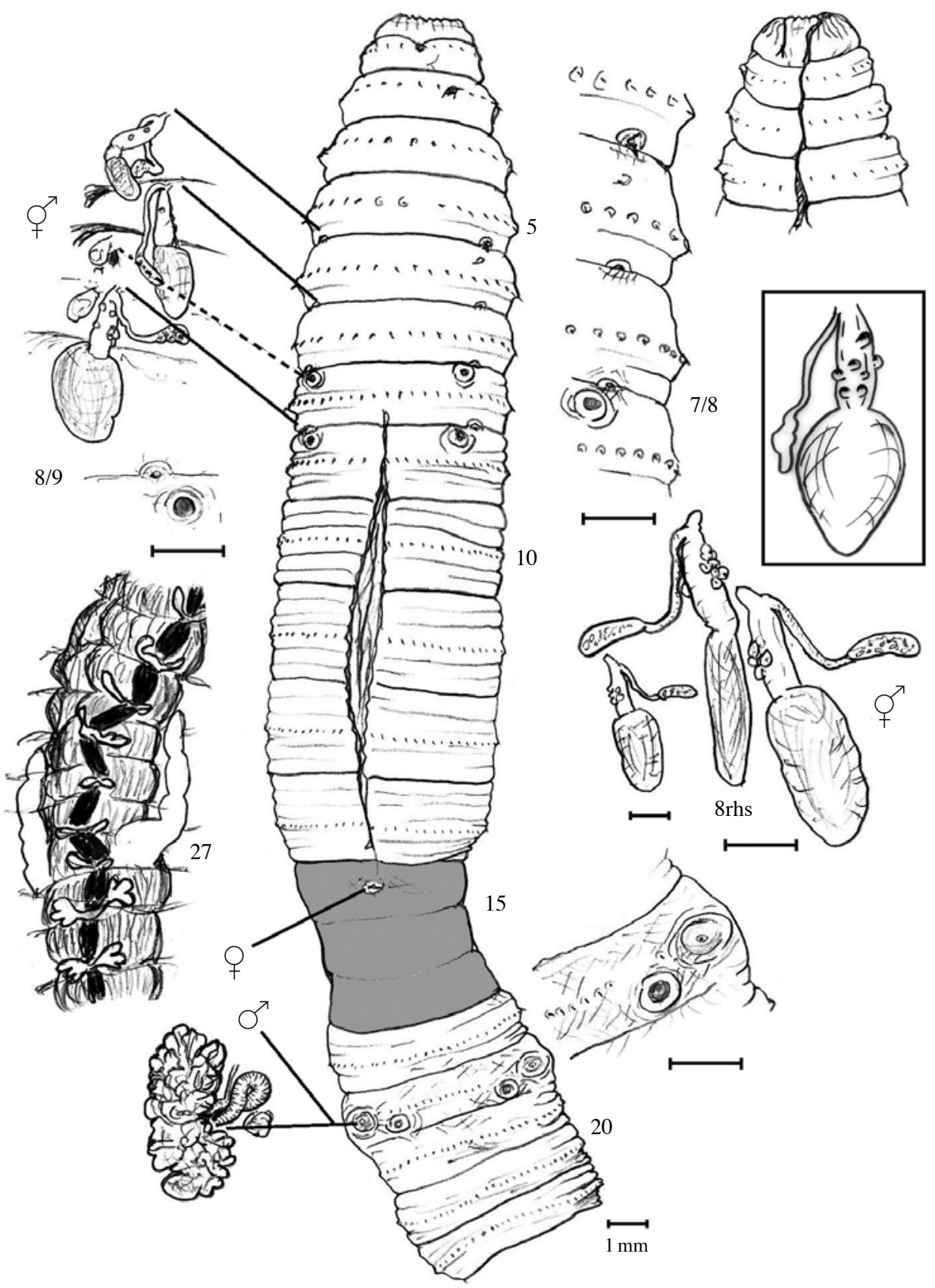

Fig. 11. Pheretima pingi previously dissected syntype (London BMNH 1924.11.29.5) showing habitus, prostomium plus spermathecae, caecum and $18 \mathrm{~h}$ s prostate in situ with enlargements of spermathecal pores and 18rhs male pore; a spermatheca with parasitic artefacts from 8rhs (removed by Stephenson and placed into a vial) figured separately at two scales and two aspects; [boxed is copy of Stephenson's sketch of same spermatheca (cf more detail in Chen, 1933: fig. 15)]. Compare to Fig. 3.

Lengths: Type $132 \mathrm{~mm}$ [for pingi Gates gave range as "140-340 mm" which was to the higher end and should perhaps be revised for carnosus to $130-340 \mathrm{~mm}$ or, when A. fornicatus was included (see below) to $78-340 \mathrm{~mm}]$. Width: ca. 4-9 mm. Segments: 126 or 127 in type. Colour: Dark slate dorsally, paler ventrally; clitellum darker; type bleached to a uniform buff in preservative (alcohol?). Prostomium: Broad closed epilobous. First dorsal pore: $11 / 12$ or $12 / 13$ (indeterminate in type as previously dissected, presumably by Stephenson, thus Gates could not be as categorical and gave range of 11/ 12-13/14). Setae: Usually 35-60 per segment (eg in the type I counted 36 on segment 5 and 60 on segment 12), those of segments 2-9 fewer but enlarged. Cf Gates 
(1939: 466) who had 17-66, but appears not to have included the count from the types. Clitellum: Annular 14-16, no setae visible. Male pores: On 18 on elliptical porophore. Female pores: Single on 14. Spermathecal pores: 5/6/7/8/9 ca, 0.3 circumference apart at posterior of translucent areas in 5-8. Cf. Gates (1939) pre-intersegmental in 5-8, this erroneous. Genital markings: Paired discs just median to lines of male and spermathecal pores (or sometimes shifted more ventrally) presetal on 8,9 (and sometimes on 18 and 19 too?); typically with a presetal pair in $8 \& 9$ and a postsetal pair on 18 .

Septa: 5/6-7/8 strong, 8/9 lacking (in type or membranous in other specimens), $9 / 10$ thin (or also lacking in other specimens), 10/11 and 11/12 considerable, 12/13 and thereafter thinning. Lymph glands dorsally on intersegments, large, paired from 15 in type (pers. obs.) or said to be from 19 by other workers. Dorsal blood vessel (dbv): Single. Hearts: 9,10-13; Gates (1939) says hearts in 10 are present except in type, this possibly wrong as from my inspection there were thin commissurals paired anteriorly in 10 just behind septum 9/10 and connecting directly to dorsal blood vessel (dbv), rather than posteriorly and connecting to $\mathrm{dbv}$ via thin constrictions that traverse posterior septa in 11-13. Gizzard: Single in 8-9. Calciferous glands: Absent; but oesophagus dilated and vascularized in type in 12 and 13 (pers. obs.). Intestine origin (caeca, typhlosole): In 15 and/or 16 (in type in 15 lhs and 16rhs) sometimes given as from 19 by other workers; caeca simple smooth from 27; thin lamellar typhlosole present from after 27 (type pers. obs.). Nephridia: Meroic. Male organs: Testes/funnels in 10 and 11 in sacs; seminal vesicles large in 11 and 12; pseudovesicles paired in 13 in type (pers. obs.). Ovaries: In 13 as usual; ovisacs small in 14 in type (pers. obs.). Prostates: Racemose glands in 17-19, duct muscular. Spermathecae: Four pairs, the ampulla oval, duct almost as long; the diverticulum from near body wall reached to half the height of ampulla with slender stalk and wider seminal chamber. Several tubercules were described on spermathecal duct walls by Stephenson, and each spermatheca in the type has these, which are probably parasitic artefacts (as also suggested by Gates, 1939: 468). Gut contents: Silty soil and coarse leafy organic remains (type, pers. obs.).

Remarks. Mistakes in Gates' (1939) redefinition of Pheretima pingi are that the setal counts are much lower than occur in the actual type (see note below on Gates' mistakes); he also mistook the septa and hearts and, quite importantly, provided genital markings that comply with A. carnosus and had spermathecal pores that he insisted were posterior in segments 5-8 but that are now shown to be in the intersegmental furrows of $5 / 6 / 7 / 8 / 9$ in the types of both A. pingi and A. carnosus.

The 'characteristic' tubercules on the spermathecal ducts are not nephridial and probably are due merely to Monocystis infestation as was also suggested by Gates (1939: 468), thus removing any justification for retaining pingi separately from A. carnosus (Goto \& Hatai, 1899). Thus its abundance in Nanking may be indicative of a possible endemic range for A. carnosus, as was suggested by Chen (1936: 275) and as concluded by Kobayashi (1936) from Korean work. Slight reticence on merging these taxa by Blakemore (2012a; 2013b) was due to supposed larger size in $A$. pingi - now known to be false as the type is only $132 \mathrm{~mm}$ long - and a later onset of intestine origin and septal glands, now also proven false. It is possible, nevertheless, that Amynthas chungkingensis with spermathecae in 6/7/8/9 (as found in two of Kobayashi's 204 Korean specimens) be retained as a separate species, this dependent on access and inspection of its types now that $A$. pingi is resolved. Gates (1939: 469) compared his concept of $A$. pingi to Pheretima diffringens (cf. A. corticis proper) and differentiated it on these points:

- Larger setal numbers (but he undercounted those in his concept of A. pingi).

- Posterior location of first dorsal pore (now known to be irrelevant and overlapping).

- Segmental location of spermathecal pores (now known to be mistaken).

- Post-clitellar GMs (irrelevant when lack of markings permissible).

- Presence of complete septum 8/9 (variable and also mistaken by Gates in the type).

Gates (1939: 436) further claimed that his $P$. fornicata Gates, 1925 from Tatsienlu, Tibet was distinguished from P. hongkongensis Michaelsen, 1910, by the dorsal gap in the setal circle of segment 2 (hardly significant), the absence of genital markings (ditto), and the exclusion of the anterior seminal vesicles from the testis sac of segment 11 (ditto). Thus $P$. fornicata possibly qualifies for synonymy of $P$. hongkongensis but the question remains: how separate are these two taxa from $A$. pingi? The former, $P$. fornicata, complies with $A$. pingi except for its lack of genital markings, i.e. it is within the ambit of Kobayashi's (1936) definition of A. carnosus. The latter, $P$. hongkongensis, also redescribed by Gates (1939: 446) from its type, differs mostly on its making near the male pores which is similar to but not explicit in Kobayashi's description of $A$. carnosus. This issue is discussed further in the next section on 'Pheretima fornicata'.

The current lectotype of $P$. pingi (=A. carnosus) then, agrees morphologically with two Hikone, Shiga specimens from Japan that provided DNA samples (JET112-114IAn460-461). The DNA of these Shiga specimens agrees $(100 \%)$ with that of Korean specimens from Jeju and the Korean mainland that provided DNA samples WO34, 
WO67, WO32, WO24, w57 (Blakemore, 2013a) and slightly less so (>99\%) for w37 and w55 samples, respectively, from Geoman and Incheon specimens. These specimens differ inconsequentially from the description given above of ' $P$. pingi' further solidifying the definition of $A$. carnosus proper that now surely includes pingi as a junior synonym.

DNA was not extractable from types of either A. carnosus nor of A. pingi. However, megaBLAST of both $A$. carnosus from Hikone (Tokyo An-460 DNA JET-112) and A. carnosus from Geoman (NIBR IV261234 DNA w37) comply $99 \%$ with A. carnosus non-type specimens from Japan (eg GenBank AB542452) and, moreover, both sequences also agree $99 \%$ (656/658) with each other by BLASTn. Since the Hikone specimen resembles (and was first though to be) A. pingi, and the Geoman specimen is the same morphologically as the A. carnosus neotype, thus both specimens provide a de facto link uniting the neotype of A. carnosus with the lectotype of A.pingi to support their merger.

Two anomalous specimens were described as "?Amynthas pingi" from Ulleungdo (Blakemore, 2013a: 60) and as "A. carnosus" from Incheon that provided DNA samples w54 and w56, respectively. While the Incheon specimen (along with a paratype from Busan) was named as Amynthas carnosus roki Blakemore, 2013 in a complementary paper in the current issue; the Ulleungdo specimen also deserving sub-specific status and is nominated above as A. carnosus naribunji.

As part of this revision of A. carnosus, it may be noted its erstwhile synonym, A. fornicatus is sometimes maintained by some Chinese workers (eg Shen et al., 2003; Wang \& Qiu, 2005; Sun et al., 2012) and it is now beholden on them to similarly clarify and define their taxonomic concepts. Nevertheless, I here briefly consider this taxon for clarity.

\section{Pheretima fornicata Gates, 1935}

(Figs. 12, 13)

Pheretima fornicata Gates, 1935: 9 figs. 5-6; Chen, 1936: 296 (re-examination of type in US National Museum); Gates, 1939: 434 (changing type locality to "Tatsienlu, Szechwan" "From Dr Graham: 3 clitellate specimens labeled 'Tatsienlu, 12,000 feet, July 7-9, 1923' and adding 1 clitellate specimen in poor condition labeled "Between Gin Keo Ho and Dawei, 1,300-5,000 feet, August 1-2".

Amynthas fornicatus: Shen et al., 2003: 482, tab. 1; Wang \& Qiu, 2005: tab. 1; Sun et al., 2012: tab. 1.

Description. The original description in full in quotes with Gates' (1939) amendments added in square braces that yet ignore Chen's (1936) additional and contradictory data from reinspection of Gates' types [data from Shen et al. (2003: tab. 1) bolded and also put in square braces but presumably not from types]:-

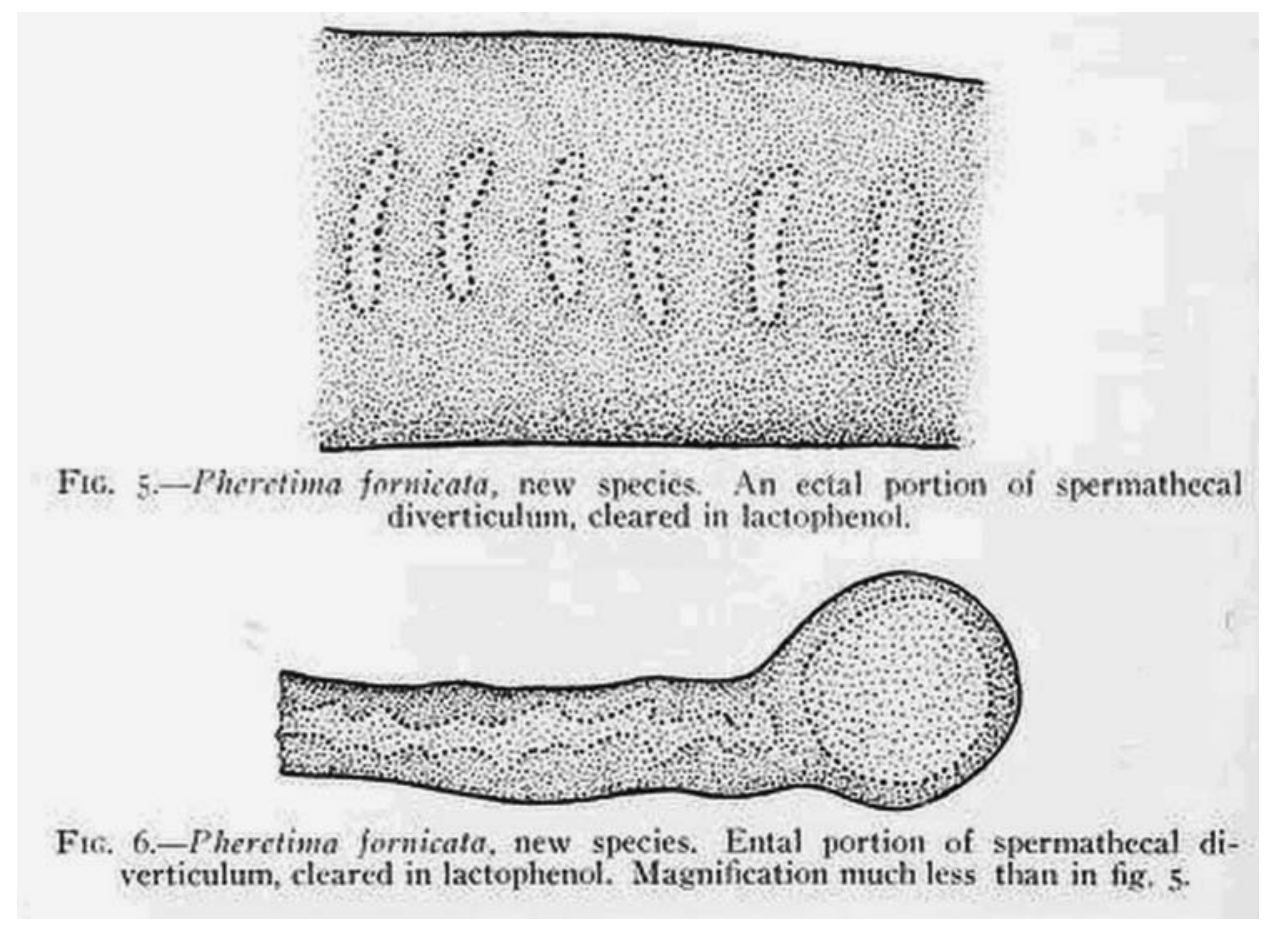

Fig. 12. Amynthas fornicatus showing Gates' (1935: figs. 5-6) most uninformative and irrelevant details of spermathecae invoking adage "missing the forest for the trees". 


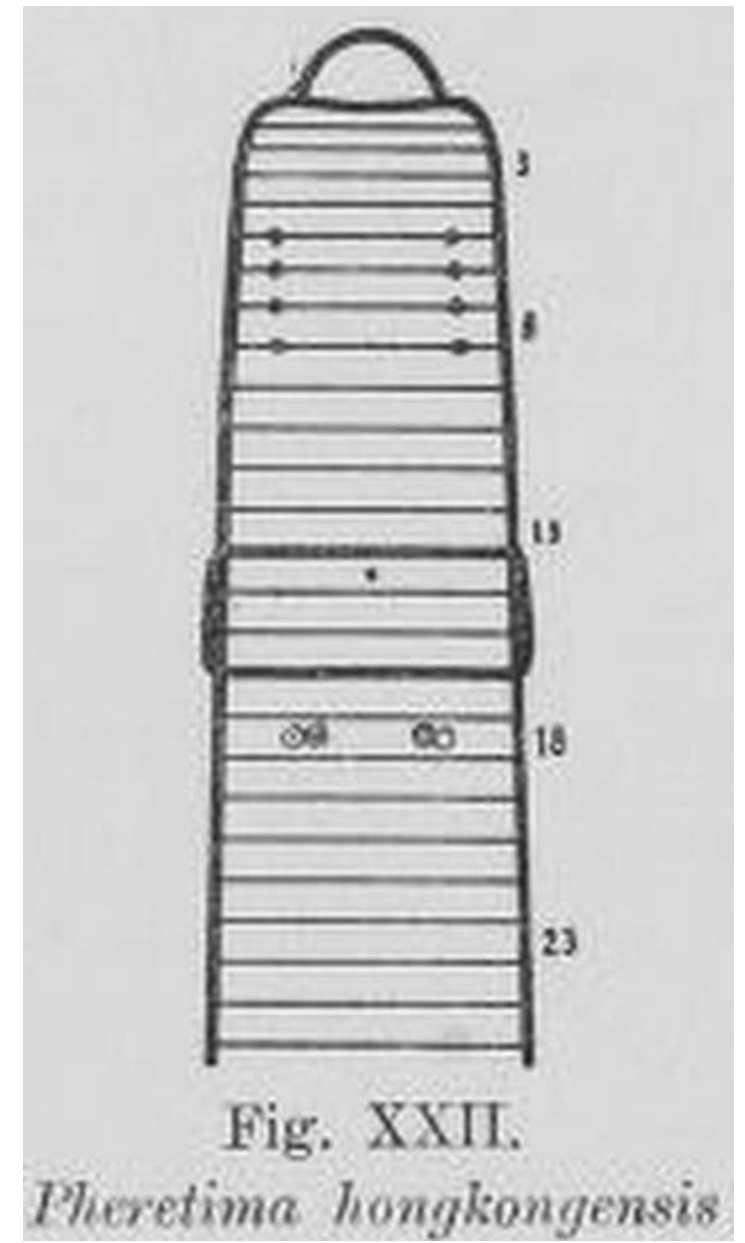

Fig. 13. Prior Amynthas hongkongensis after Michaelsen (1910: fig. 22), for comparison.

"PHERETIMA FORNICATA, n. sp. Length 78 to 90 $\mathrm{mm}$ [100 mm], diameter 4 to $6 \mathrm{~mm}$. [Segments 90-105]. Setae: vi/17-24 [16-26] [43-50], vii/19-21 [19-23], viii/ 18-23 [18-27] [42-52], [xii/42-52], xvii/13-14 [13-19], xviii/9-14, xix/12-15, [x, 56,] xx/56, [xxv 45-55]; a wide dorsal gap in the setal circle of ii. First dorsal pore in 12/ 13 [with pore like marking in 11/12]. [Clitellum annular, 13/14-16/17]. Spermathecal pores minute and superficial, four pairs, in 5/6-8/9 [on tiny, grayish, transversely oval markings]. Male pores superficial, on circular to transversely oval, disk-shaped porophores [that are about 0.5 mm wide]. No genital markings. Septum $8 / 9$ present but membranous [9/10 laking]. [Intestine from 15]. Intestinal caeca simple. [Hearts in 9-13 to ventral blood vessel, those in 10 and 11 within testis sac]. Testis sacs of $\mathrm{x}$ and $\mathrm{xi}$ unpaired and horseshoe-shaped. [Seminal vesicles are in $11 \& 12$, pseudovesicles in 13 and sometimes 14]. [Spermathecal duct as long as the ampulla, diverticulum longer than duct and ampulla]. Spermathecal diverticulum with a long, slender stalk and a spheroidal or asymmetrical seminal chamber. Type. - U.S.N.M no. 200099, from Tatsienlu, Tibet.

Distinguished from $P$. hongkongensis by the gap in the setal circle of ii, the exclusion of the seminal vesicles of xi from the posterior testis sac, and the absence of genital markings." [None of these latter three characters seems sufficient justification for their separation in context with the current revisions of A. carnosus].

Remarks. Chen (1936: 298) said P. fornicata "is probably identical with $P$. pingi Steph.” but Gates (1939: 436) responded tersely with " $P$. fornicata is, on the contrary, clearly distinguished from $P$. ping $i$ by the horseshoeshaped testis sacs of $\mathrm{x}$ and xi." However, I think this is an unreliable character for separation and am obliged to combine them.

Gates (1935) had implicated his fornicata as being closest to P. hongkongensis Michaelsen, 1910: 107 based on a probable immature and abnormal type that has only a pair of small marking immediately median to male pores on 18 which is all that separates it from A. carnosus as redescribed by Blakemore (2012a) based on its neotype, and on Kobayashi's (1936) excellent redescription in a publication that Gates (1939: 469) was clearly aware of but rejected for unsound reasons.

Thus differences of $P$. fornicata from $A$. pingi $(=A$. carnosus) as reinspected here from its type, are mainly a lack of genital markings which is irrelevant for A. carnosus, and the uncertainty about spermathecal papillae. However, the name fornicata is complicit with the redescription of $A$. carnosus based on its neotype by Blakemore (2012d) and on subsequent records: in particular it agrees with Kobayashi's (1936) absent GMs form types I \& I. Thus fornicata should probably be included in ping $i$ as Chen suggested and both now seemingly merit inclusion in synonymy of the prior A. carnosus (Goto \& Hatai, 1899).

Another recently described taxon, Amynthas taiwumontis Shen et al. (2013) was compared by those authors to A. fornicatus. However its description appears to comply with A. carnosus having Kobayashi's spermathecal pores type I or II and male pores type I, as redescribed by Blakemore (2012a) (this overlooked despite my sending a copy to Dr Huei-Ping Shen) and thus this name too is another probable inclusion in the long synonym list of A. carnosus presented in the Abstract above.

However, any of these specimens with spermathecal pores in 5/6/7/8/9 not on papillae/porophores described for $A$. carnosus probably equate more to A. corticis, as indeed does $A$. penpuensis Shen et al., 2003 as syn. nov. since all that currently separates it is its dorsal pores slightly more in advance (from $5 / 6$ or $6 / 7$ rather than from $11 / 12$ abouts), this single character is doubtfully adequate for species level separation. 


\section{Notes on Gates' taxonomic mistakes and the consequences of these.}

Works just quoted shows how Gates (1939) was mistaken - as I long suspected he was inconsistently guilty of - in just counting setae on one side for some of his data tables with "setal numbers" in different segments, whereas these were corrected to full counts by Chen (1936) and Shen et al. (2003) as is normal and proper to do. This point in itself is important as it seems Gates was inconsistent in often publishing with only half the number of setae in only some of his taxa, thereby making his species comparisons both confusing as well as wrong. This needs to be resolved from all his types that remain and other inspected materials, currently beyond the scope of the current work. He often also intermixed synonyms with citations without punctuation.

Moreover, Gates (1939: 415 - said: "Intestinal caeca characterized by a single longitudinal axis are termed simple regardless of the depth of incisions on the dorsal and (or) ventral margins of the primary caecal evagination. Intestinal caeca with several longitudinal axes and with a rather characteristic glove-shaped conformation are termed compound." Thus, for unfathomable logic, he deliberately ignores the relatively useful feature of incised caeca making his work again suspect. Instead of any useful figures, he offers us irrelevant details of parts of a diverticulum duct, but usually no figures at all. Perhaps the worst mistake by Gates $(1972 ; 1982)$ was deliberately ignoring profound revision of all pheretimoids by Sims \& Easton (1972) that he was clearly aware of, and thus allowing novices to persist with defunct Pheretima Kinberg, 1867: 97, 102 as default genus for decades rather than accept proper prior Amynthas Kinberg, 1867: 97, 101.

\section{Taxonomic 'Housekeeping'}

\section{A. Replacement of homomym and corrections of critical errors in Sun et al. (2012).}

Page 149. Amynthas trapezoides Qiu \& Sun, 2010 is mispelt "trapezoidesi".

Page 152. A. fuscus Qiu \& Sun, 2012 is a junior synonym of A. carnosus (Goto \& Hatai, 1899), Kobayashi's (1936) Type I \& I as redescribed by Blakemore (2012a: fig. 3).

Page 153. A. fornicatus (Gates, 1935) was maintained (Qiu et al., 2012: 153) (vide infra).

Page 154. Amynthas montanus Qiu \& Sun, 2012 is a secondary homonym of Pheretima montana Ishizuka, 1999 (non Kinberg, 1867) as defined under ICZN (1999: Arts. 52-60), but a replacement name was not automatically provided as it appeared to the current author to be similar to, if not a junior synonym of, A. micronarius (Goto \& Hatai, 1898) as redescribed by Blakemore (2012c). The original authors (pers. comm. RJB Sept, 2012) were notified and accepted anomalies in their description: For example, the setae between its male pores are stated (Qiu et al., 2012: 154, tabs. 2-3) to number "12-18", almost as few as in A. micronarius, yet clearly shown (Qiu et al., 2012: fig 2) to be much greater (ca. 26 setae). Molecular data may be sought to confirm its relationships, in the meantime, since there is a difference of taxonomic judgment and it is proposed to provisionally retain it as a valid taxon, then its name montanus must be rejected and replaced using procedures described in ICZN (1999: Art. 60). Thus, with agreement of authors (pers. com. $3^{\text {rd }}$ Aug., 2013) A. montanus Qiu \& Sun, 2012 is hereby given a new substitute name as Amynthas zhuya Blakemore nom. nov. (from an historical epithet for Hainan of Pearl Cliffs - 珠崖 or Zhūyá) having the same type and characters as originally.

\section{B. Replacement name for sp. nov. misdescribed by Shen et al. (2005) as A. carnosus.}

Shen et al. (2005) claimed A. carnosus from Taiwan, but their description was based on some other entity that appears to be a new taxon here provided a replacement name as Amynthas yizhou sp. nov. It is similarly octothecal with spermathecal pores in 5/6/7/8/9 but differs from A. carnosus (Goto \& Hatai, 1899) with regards to its GMs; in particular, it has two or three small papillae placed just ventral and on either side of the intersegmental furrow near each spermathecal pore plus a pair of papillae directly above and below the male pores. On these characters it is separable from $A$. carnosus described by Blakemore (2012a) and herein. Other characteristics are as per Shen et al. (2005, fig. 1) based on 23 specimens (=syntypes, deposited in the Endemic Species Research Institute, Chichi, Nantou, Taiwan); the holotype being the one figured. The name is derived from an historical Chinese term for the island, Yizhou (夷洲).

\section{ACKNOWLedgements}

The Korean biodiversity project under auspices Prof. Wonchoel Lee (Hanyang Uni) was supported by NIBR and funded by the Ministry of Environment (MOE) of Republic of Korea. Families of my sisters, Annette Hill and the Jill Davey, are thanked for accommodating this research in the UK. In Canada, Sean Prosser and Natalie Ivanova of Guelph Uni Barcoding Centre attempted DNA analyses under the direction of Dr Paul Hebert. Thanks for loans and curation of museum types to staff at NIBR (Incheon), Dr Tom Trnski (Auckland), Emma Sherlock (NHM, London), Dr T. Kuramochi (Tokyo). JSR editors and referees are thanked for constructive comments that improved first drafts. A Chinese proverb says "The beginning of wisdom is to call a thing by its correct name", 
in this regard I am grateful for the contributions and understanding of Chinese colleagues.

\section{REFERENCES}

\section{(Not all authorities forming part of an ICZN binomial cited here)}

Baird, W. 1869a. Description of a new Species of Earth-worm (Megascolex diffringens) found in North Wales. Proceedings of the general meeting for scientific business of the Zoological Society of London 1869:40-43 [Available from: http://www.archive.org/stream/proceedingsofgen 69zool\#page/40/mode/2up].

Baird, W. 1869b. Additional Remarks on the Megascolex diffringens. Proceedings of the general meeting for scientific business of the Zoological Society of London 1869:387-389.

Beddard, F.E. 1892. On some Perichaetidae from Japan. Zoologische Jahrbücher, Abteilung für Systematik 6: 755-766 [Available from: http://biostor.org/reference/ $14791]$.

Beddard, F.E. 1900. Earthworm of the Hawaiian Archipelago. In: Fauna Hawaiiensis, David Sharp, Ed. (Cambridge Univ. Press) 2(1):413-426 [Available from: hbs. bishopmuseum.org/pubs-online/pdf/fh2-4annelida.pdf].

Blakemore, R.J. 2003. Japanese Earthworms (Annelida: Oligochaeta): a Review and Checklist of Species. Organisms, Diversity and Evolution 3(3):241-244 [Available from: http://www.urbanfischer.de/journals/ode/Electronic Supplement 2003-11 http://www.senckenberg.de/odes/ 03-11.htm].

Blakemore, R.J. 2008. An updated checklist of pheretimoids (e.g. Amynthas, Duplodicodrilus, Metaphire, Pheretima, Polypheretima, etc.). In: A Series of Searchable Texts on Earthworm Biodiversity, Ecology and Systematics. [CD publication, Available from: http://www.annelida.net/ earthworm/Pheretimoids.pdf].

Blakemore, R.J. 2012a. Amynthas carnosus (Goto \& Hatai, 1899) redescribed on its neotype (Oligochaeta: Megadrilacea: Megascolecidae). Journal of Species Research 1(1):35-43 [Available from: www.nibr.go.kr/download? attach_path=journal\&attach $=2012$ vol1no1_4.pdf].

Blakemore, R.J. 2012b. Earthworms from NIBR Jeju-do biosphere compared to historical and new Japanese types (Oligochaeta: Megadrilacea: Megascolecidae). Journal of Species Research 1(2):133-115 [Available from: www. nibr.go.kr/ english/event/journal_spe_3.jsp].

Blakemore, R.J. 2012c. On opening a box of worms (Oligochaeta, Megascolecidae) - Historical earthworm specimens transferred to Tokyo from the Saito Ho-on Museum of Natural History in Sendai. Bulletin of the National Science Museum, Tokyo Series A 38(3):95-124.

Blakemore, R.J. 2012d. Cosmopolitan earthworms. $5^{\text {th }}$ Edi- tion. VermEcology, Yokohama, Japan. pp. 950 [CD publication].

Blakemore, R.J. 2013a. Ulleung-do earthworms - Dagelet Island revisited. Journal of Species Research 2(1):55-69 [Available from: www.nibr.go.kr/eng/event/journal_ spe_3.jsp].

Blakemore, R.J. 2013b. Jeju-do earthworms (Oligochaeta: Megadrilacea)-Quelpart Island revisited. Journal of Species Research 2(1):15-54 [Available from: www.nibr.go. $\mathrm{kr} / \mathrm{eng}$ /event/ journal_spe_3.jsp].

Blakemore, R.J. and R. Ueshima. 2011. Catalogue of Annelida: Oligochaeta in University of Tokyo Zoology Museum (ZMUT). In: R. Ushima (Ed.), Catalogue of Invertebrate Collection Deposited in the Department of Zoology, the University Museum, the University of Tokyo (3), Phylum Annelida (Class Polychaeta, Oligochaeta, and Hirudinida). Material Reports 90:59-83 [Available from: http://umdb. um.u-tokyo.ac.jp/DDoubutu/invertebrate_en/index.html].

Blakemore, R.J. and S. Lee. 2013. Preliminary survey of Busan earthworm taxa supported by DNA barcodes. Journal of Species Research 2(2):127-144.

Blakemore, R.J., E. Kupriyanova and M.J. Grygier. 2010. Neotypification of Drawida hattamimizu Hatai, 1930 (Oligochaeta: Megadrili: Moniligastridae) and the first COI sequence from an earthworm type. ZooKeys 41:129 [Available from: http://pensoftonline.net/zookeys/ index.php/journal/article/view/374/401].

Blakemore, R.J., T.-S. Park and H.-Y. Seo. 2012. A new Korean earthworm (Oligochaeta: Megadrilacea: Megascolecidae). Zootaxa 3368:256-262 [Available from: http:// www.mapress.com/zootaxa/2012/f/zt03368p262.pdf].

Chang, C.-H., H.-P. Shen and J.-H. Chen. 2009. Earthworm fauna of Taiwan. National University Press, Taipei. pp. 167.

Chen, Y. 1933. A preliminary survey of the earthworms of the Lower Yangtze Valley. Contributions from the Biological Laboratory of the Science Society of China 9(6): 177-296.

Chen, Y. 1936. On the terrestrial Oligochaeta from Szechuan II with the notes on Gates' types. Contribibutions of Biological Laboratory of Science Society of China (Zoology) 11:269-306.

Chen, Y. 1959. Chinese Animal Atlas. Annelida. Science Press, Beijing. pp. 29 [in Chinese].

Easton, E.G. 1979. A revision of the 'acaecate' earthworms of the Pheretima group (Megascolecidae: Oligochaeta): Archipheretima, Metapheretima, Planapheretima, Pleionogaster and Polypheretima. Bulletin of the British Museum (Natural History) Zoology 35(1):1-128.

Easton, E.G. 1981. Japanese earthworms: a synopsis of the Megadrile species. Bulletin of the British Museum (Natural History) Zoology 40:33-65.

Easton, E.G. 1982. Australian pheretimoid earthworms (Megascolecidae: Oligochaeta): a synopsis with the descrip- 
tion of a new genus and five new species. Australian Journal of Zoology 30:711-735.

Easton, E.G. 1984. Earthworms (Oligochaeta) from islands of the south-western Pacific, and a note on two species from Papua New Guinea. New Zealand Journal of Zoology 11:111-128.

Fletcher, J.J. 1886. Notes on Australian Earthworms. Part I. Proceedings of the Linnean Society of NSW (2)1:523576 [Available from: http://www.archive.org/stream/ proceeding soflinn0201linn\#page/n3/mode/2up].

Gates, G.E. 1935. New earthworms from China, with notes on the synonymy of some Chinese species of Drawida and Pheretima. Smithsonian Miscellaneous Collections 93:1-19.

Gates, G.E. 1939. On some species of Chinese earthworms with special reference to specimens collected in Szechuan by Dr. D.C. Graham. Proceedings of the United States National Museum 85:405-507 [Available from: si-dppr. si.edu/dspace/bitstream/10088/16290/USNMP-85_3040 _1939. pdf].

Gates, G.E. 1943. On some American and Oriental earthworms. Ohio Journal of Science 43:87-116.

Gates, G.E. 1972. Burmese Earthworms, an introduction to the systematics and biology of Megadrile oligochaetes with special reference to South-East Asia. Transactions of the American Philosophical Society 62(7):1-326. [Available from: http://www.jstor.org/stable/1006214 in 2011].

Gates, G.E. 1982. Farewell to North American megadriles. Megadrilogica 4:12-77.

Goto, S. and S. Hatai. 1898. New or imperfectly known species of earthworms. No. 1. Annotations Zoologicae Japonensis 2:65-78.

Goto, S. and S. Hatai. 1899. New or imperfectly known species of earthworms. No. 2. Annotations Zoologicae Japonensis 3(1):13-24.

Hong, Y. and S.W. James. 2001. Five new earthworms of the genus Amynthas Kinberg (Megascolecidae) with four pairs of spermathecae. Zoological Studies 40(4):269-275.

Hong, Y. and T.-H. Kim. 2009. The earthworm composition in agroecosystem of Sunyu Island, Korea. The Korean Journal of Environmental Biology 27(2):135-139.

ICZN (1999). International Code of Zoological Nomenclature ( $4^{\text {th }}$ edition). International Trust for Zoological Nomenclature, c/o Natural History Museum, London. pp. 306 [Available from: http://www.iczn.org/iczn/index.jsp].

Ishizuka, K. 2001. Taxonomic Study of the Genus Pheretima s. lat (Oligochaeta, Megascolecidae) from Japan. Bulletin of Seikei University 33(3):1-125.

Kinberg, J.G.H. 1867. Annulata nova. Ofersigt af Kongl. Vetenskaos-Akademiens Förhandlingar, Stockholm 23(4):97-103.

Kobayashi, S. 1934. Three new Korean earthworms belonging to the genus Pheretima, together with the wider range of the distribution of Pheretima hilgendorfi (Michaelsen). Journal of Chosen Natural History Society 19:1-11

Kobayashi, S. 1936. Distribution and some external characteristics of Pheretima $(P h$.) carnosa (Goto et Hatai) from Korea. Science Report of the Tohoku Imperial University 11(1):115-138.

Kobayashi, S. 1937. Preliminary survey of the earthworms of Quelpart Island. Science Report of the Tohoku Imperial University (B) 11(3):333-351.

Kobayashi, S. 1938. Earthworms of Korea I. Science Report of the Tohoku Imperial University 13(2):89-170.

Michaelsen, W. 1892. Terricolen der Berliner Zoologischen Sammlung. II. Archiv fur Naturgeschichte. Berlin 58:209261 [Available from: http://biostor.org/reference/61378].

Michaelsen, W. 1900. Das Tierreich Vol. 10: Vermes, Oligochaeta. Friedländer \& Sohn, Berlin. pp. 575 [Available from: http://www.archive.org/details/oligochaeta10mich].

Ohfuchi, S. 1937. On the species possessing four pairs of spermathecae in the genus Pheretima, together with the variability of some external and internal characteristics. Saito Ho-On Kai Museum Research Bulletin 12:31-136.

Shen, H.-P., C.-F. Tsai and S.-C. Tsai. 2003. Six new earthworms of the genus Amynthas (Oligochaeta: Megascolecidae) from Central Taiwan. Zoolological Studies 42(4): 479-490 [Available from://zoolstud.sinica.edu.tw/Journals/ 42.4/ 479.pdf].

Shen, H.-P., S.-C. Tsai, C.-F. Tsai and J.-H. Chen. 2005. Occurrence of the Earthworm Amynthas carnosus (Goto and Hatai, 1899) in the Northern Taiwan. Endemic Species Research 7(1):95-100 [Available from: https://tesri. coa.gov.tw/files/tesri_protect/124/070108.pdf].

Shen, H.-P., C.-H. Chang, C.-L. Li, W.-J. Chih and J.-H. Chen. 2013. Four new earthworms species of the genus Amynthas (Oligochaeta: Megascolecidae) from Kinmen, Taiwan. Zootaxa 3599:471-482.

Sims, R.W. and E.G. Easton. 1972. A numerical revision of the earthworm genus Pheretima auct. (Megascolecidae: Oligochaeta) with the recognition of new genera and an appendix on the earthworms collected by the Royal Society North Borneo Expedition. Biological Journal of the Linnean Society 4:169-268.

Sims, R.W. and B.M. Gerard. 1985. Earthworms. Keys and notes to the identification and study of the Species. Synopsis of the British Fauna (New series). E.J. Brill, Leiden. No. 31. pp. 171.

Song, M.J. and K.Y. Paik. 1969. Supplemental note of Pheretima kanrazana and Ph. soulensis. Theses Colloquium Communication on $60^{\text {th }}$ Birthday of Dr In Suck Yang. pp. 135-144.

Stephenson, J. 1925. Oligochaeta from various regions, including those collected by the Mount Everest Expedition 1924. Proceedings of the Zoological Society of London 95(3): 879-907.

Stephenson, J. 1931. Oligochaeta from Burma, Kenya and 
other parts of the world. Proceedings of the Zoological Society of London 1931:33-92.

Sun, J., Q. Zhao and J.-P. Qiu. 2012. Four new species of the Amynthas corticis-group (Oligochaeta: Megascolecidae) from Hainan Island, China. Zootaxa 2680:149-158.

Vaillant, M.I. 1872. On the acclimatization and anatomy of Perichata diffringens, Baird. Annals and Magazine of Natural History 1872:322-324 [Available from: http:// biostor.org/reference/92031].

Wang, H.-J. and J.-P. Qiu. 2005. A Preliminary Report on
Pheretimoid Earthworms from the Xingdou Mountain Nature Reserve-With Description of Two New Species and a New Subspecies of the Genera Amynthas and Metaphire. Journal of Shanghai JIaotong University (Agricultural Science) 23(1):23-30 [In Chinese with English summary, Available from: http://file.lw23.com/0/00/00a/ 00a16e20-280a-4e0a-b495-c0b857805d8b.pdf].

Submitted: June 7, 2013, Accepted: August 12, 2013 


\section{Appendix of DNA data (BLAST from http://blast.ncbi.nlm.nih.gov/Blast.cgi)}

[WMxx, WOyy and wzz samples are from Korean studies; JET samples are the author's iBOL (www.boldsystems.org) project on Japanese Earthworms. Data used for maximum likelihood phylotree from MEGA5 (using program defaults with samples WM7, WM9, WO4-6 and GenBank DQ257319 Complements Reversed for ClustalW alignment)].

> WM7 A. corticis from Arataki Honey, Waikato New Zealand (RJB) - AMNZ-86033.

GGTGTTGATATAAAATTGGGTCTCCCCCTCCTGCTGGATCAAAGAATGATGTATTAAGGTTTCGATCTGTTAATAGTATTGTG ATAGCACCGGCTAGTACTGGTAATGATAGAAGTAGTAGAACTACGGTAATTACTACTGCTCATACAAATAGGGGAATTCGT TCTAGTCGTAGGCCTGATCATCGTATATTAATTACTGTAGTAATAAAATTGATTGCACCTAGAATTGATGATGCCCCTGCTAA GTGTAGTGAGAAAATTGCCAGATCTACTGATGGTCCAGCATGCGCAATGTTACTTGCTAGTGGTGGGTAAACTGTTCATCCT GTTCCTGCACCTTTTTCCACTGCTGCAGAAGAGACTAGTAAGATGAGTGAGGGGGGTAATAGTCAGAATCTTATATTATTTA GGCGTGGAAATGCTATATCTGGAGTCCCCAATATAAGTGGTAATAGTCAATTACCAAAACCACCAATAAATACTGGTATTAC TAGAAAAAAAATTATTAAGAATGCATGTGCTGTTACAATTGTGTTATAAAGTTGGTCACTTCCCAGGAATGACCCAGGTTGT CTTAATTCGATTCGAATAAGAAGACTTATTCCAGCCCCAATTATTCCGGCTCAAATTCCTAAAATGAAGTATA megaBLAST Maximum Id=100\% DQ224190 Amynthas corticis voucher, Taiwan.

> WM9 Korea $1^{\text {st }}$ Survey site \#3 A. corticis dissected (RJB) - NIBRIV249903. AGATGTTGATATAAAATTGGGTCCCCCCCTCCCCGCAGGATCAAAGAATGATGTATTAAGGTTTCGATCTGTTAATAGTATT GTAATAGCACCTGCTAGTACTGGTAATGATATAAGTAGTAAAACTGTGGTGATTACCACTGCTCATACAAATAGGGGAATTC GTTCCAGTTGTAGCCCGGATCATCGTATATTAATTACTGTAGTAATAAAATTGATTGCACCTAGAATTGATGATGCCCCCGCT AAGTGTAGTGAGAAAATTGCTAGATCTACTGATGGTCCAGCATGTGCAATATTACTTGCTAGTGGGGGATATACTGTTCATC CTGTTCCCGCACCCTTTTCTACTGCTGCAGAAGAGACTAATAGGATGAGCGATGGAGGCAATAGTCAGAATCTTATATTATT TAGGCGTGGGAATGCTATATCTGGAGTTCCTAATATAAGTGGTAATAATCAGTTACCAAAGCCACCAATAAATACTGGTATT ACTAGAAAAAAAATTATTAAAAATGCATGTGCTGTTACAATTGTGTTATATAGTTGGTCACTTCCCAGGAATGATCCTGGTT GTCTTAATTCGATTCGAATAAGAAGACTTATTCCTGCCCCAATTATTCCGGCTCAAATTCCTAAAATGAAGTATAGGG megaBLAST Max Id=99\% JX290433.1I Amynthas corticis voucher, Taiwan.

> WO4 Korea $1^{\text {st }}$ Survey site \#4 Temple worm 1 Korea (RJB) - NIBRIV249906. AATAGATGTTGATATAAAATTGGGTCCCCCCCTCCCGCAGGATCAAAGAATGATGTATTAAGGTTTCGATCTGTTAATAGTA TTGTAATAGCACCTGCTAGTACTGGTAATGATATAAGTAGTAAAACTGTGGTGATTACCACTGCTCATACAAATAGGGGAAT TCGTTCCAGTTGTAGCCCGGATCATCGTATATTAATTACTGTAGTAATAAAATTGATTGCACCTAGAATTGATGATGCCCCCG CTAAGTGTAGTGAGAAAATTGCTAGATCTACTGATGGTCCAGCATGTGCAATATTACTTGCTAGTGGGGGATATACTGTTCA TCCTGTTCCCGCACCCTTTTCTACTGCTGCAGAAGAGACTAATAGGATGAGCGATGGAGGCAATAGTCAGAATCTTATATTA TTTAGGCGTGGGAATGCTATATCTGGAGTTCCTAATATAAGTGGTAATAATCAGTTACCAAAGCCACCAATAAATACTGGTA TTACTAGAAAAAAAATTATTAAAAATGCATGTGCTGTTACAATTGTGTTATATAGTTGGTCACTTCCCAGGAATGATCCTGG TTGTCTTAATTCGATTCGAATAAGAAGACTTATTCCTGCCCCAATTATTCCGGCTCAAATTCCTAAAATGAAGTATAGGGTTCC megaBLAST Max Id=100\% JX290433.1I Amynthas corticis voucher, Taiwan.

$>$ WO5 Korea $1^{\text {st }}$ Survey site \#4 Temple worm $2=$ A. corticis ditto WO4 -IV249907. AGATGTTGATATAAAATTGGGTCCCCCCCTCCCGCAGGATCAAAGAATGATGTATTAAGGTTTCGATCTGTTAATAGTATTG TAATAGCACCTGCTAGTACTGGTAATGATATAAGTAGTAAAACTGTGGTGATTACCACTGCTCATACAAATAGGGGAATTCG TTCCAGTTGTAGCCCGGATCATCGTATATTAATTACTGTAGTAATAAAATTGATTGCACCTAGAATTGATGATGCCCCCGCTA AGTGTAGTGAGAAAATTGCTAGATCTACTGATGGTCCAGCATGTGCAATATTACTTGCTAGTGGGGGATATACTGTTCATCC TGTTCCCGCACCCTTTTCTACTGCTGCAGAAGAGACTAATAGGATGAGCGATGGAGGCAATAGTCAGAATCTTATATTATTT AGGCGTGGGAATGCTATATCTGGAGTTCCTAATATAAGTGGTAATAATCAGTTACCAAAGCCACCAATAAATACTGGTATTA CTAGAAAAAAAATTATTAAAAATGCATGTGCTGTTACAATTGTGTTATATAGTTGGTCACTTCCCAGGAATGATCCTGGTTG TCTTAATTCGATTCGAATAAGAAGACTTATTCCTGCCCCAATTATTCCGGCTCAAATTCCTAAAATGAAGT nBLAST WO4 vs. WO5 Identities=646/646 (100\%), Gaps=0/646 (0\%).

> WO6 Korea $1^{\text {st }}$ Survey site \#4 Temple worm 3=A. corticis ditto WO4 - IV249908. AGATGTTGATATAAAATTGGGACCCCCCCTCCCGCAGGATCAAAGAATGATGTATTAAGGTTTCGATCTGTTAATAGTATTG TAATAGCACCTGCTAGTACTGGTAATGATATAAGTAGTAAAACTGTGGTGATTACCACTGCTCATACAAATAGGGGAATTCG TTCCAGTTGTAGCCCGGATCATCGTATATTAATTACTGTAGTAATAAAATTGATTGCACCTAGAATTGATGATGCCCCCGCTA AGTGTAGTGAGAAAATTGCTAGATCTACTGATGGTCCAGCATGTGCAATATTACTTGCTAGTGGGGGATATACTGTTCATCC TGTTCCCGCACCCTTTTCTACTGCTGCAGAAGAGACTAATAGGATGAGCGATGGAGGCAATAGTCAGAATCTTATATTATTT AGGCGTGGGAATGCTATATCTGGAGTTCCTAATATAAGTGGTAATAATCAGTTACCAAAGCCACCAATAAATACTGGTATTA 
CTAGAAAAAAAATTATTAAAAATGCATGTGCTGTTACAATTGTGTTATATAGTTGGTCACTTCCCAGGAATGATCCTGGTTG TCTTAATTCGATTCGAATAAGAAGACTTATTCCTGCCCCAATTATTCCGGCTCAAATTCCTAAAATGAAGTATAGG nBLAST WO6 vs WO4 Identities $=650 / 651(99 \%)$, Gaps $=0 / 651(0 \%)-$ OK.

$>$ WO24 NIBR $3^{\text {rd }}$ April Amynthas carnosus? from Incheon NIBR. TTTATATTTTATCTTGGGAATCTGGGCAGGAATAATTGGTGCCGGTATGAGACTACTTATTCGAATCGAGCTCAGGCAACCG GGCTCCTTTCTGGGAAGGGACCAACTATATAACACAATTGTAACAGCACACGCATTCCTTATAATTTTCTTTCTAGTTATACC AGTATTCATTGGGGGATTTGGAAATTGGCTACTACCACTCATACTGGGAACACCGGACATAGCATTTCCACGCCTAAACAAC ATGAGATTCTGGCTACTGCCCCCATCACTAATCTTACTAGTATCTTCCGCCGCAGTAGAGAAAGGAGCAGGTACAGGATGAA CAGTATATCCCCCCCTAGCAAGAAATATTGCTCATGCGGGGCCATCAGTAGACCTGGCAATTTTCTCACTCCACTTAGCTGG GGCATCCTCAATTTTGGGGGCCATTAACTTTATTACTACAGTAATTAATATGCGCTGGTCTGGACTACGTCTAGAGCGAATC CCGCTATTTGTATGAGCAGTAGTAATTACTGTCGTACTACTATTACTATCATTACCAGTTCTTGCCGGAGCTATTACAATACT ACTAACAGACCGAAACCTAAATACATCATTCTTCGACCCAGCGGGAGGGGGGGACCCAATTCTGTACCAACACCTATTTTG megaBLAST A. carnosus from Japan 99\% Identities=640/641 (99\%), Gaps=0/641 (0\%).

> WO32 Sammock $1^{\text {st }}$ May, 2012 Amynthas carnosus. TTTATATTTTATCTTGGGAATCTGGGCAGGAATAATTGGTGCCGGTATGAGACTACTTATTCGAATCGAGCTCAGGCAACCG GGCTCCTTTCTGGGAAGGGACCAACTATATAACACAATTGTAACAGCACACGCATTCCTTATAATTTTCTTTCTAGTTATACC AGTATTCATTGGGGGATTTGGAAATTGGCTACTACCACTCATACTGGGAACACCGGACATAGCATTTCCACGCCTAAACAAC ATGAGATTCTGGCTACTGCCCCCATCACTAATCTTACTAGTATCTTCCGCCGCAGTAGAGAAAGGAGCAGGTACAGGATGAA CAGTATATCCCCCCCTAGCAAGAAATATTGCTCATGCGGGGCCATCAGTAGACCTGGCAATTTTCTCACTCCACTTAGCTGG GGCATCCTCAATTTTGGGGGCCATTAACTTTATTACTACAGTAATTAATATGCGCTGGTCTGGACTACGTCTAGAGCGAATC CCGCTATTTGTATGAGCAGTAGTAATTACTGTCGTACTACTATTACTATCATTACCAGTTCTTGCCGGAGCTATTACAATACT ACTAACAGACCGAAACCTAAATACATCATTCTTCGACCCAGCGGGAGGGGGGGACCCAATTCTGTACCAACACCTATTT megaBLAST - result 99\% GenBank A. carnosus.

$>$ WO34 Sido $2^{\text {nd }}$ May, 2012.

TTATATTTTATCTTGGGAATCTGGGCAGGAATAATTGGTGCCGGTATGAGACTACTTATTCGAATCGAGCTCAGGCAACCGG GCTCCTTTCTGGGAAGGGACCAACTATATAACACAATTGTAACAGCACACGCATTCCTTATAATTTTCTTTCTAGTTATACCA GTATTCATTGGGGGATTTGGAAATTGGCTACTACCACTCATACTGGGAACACCGGACATAGCATTTCCACGCCTAAACAACA TGAGATTCTGGCTACTGCCCCCATCACTAATCTTACTAGTATCTTCCGCCGCAGTAGAGAAAGGAGCAGGTACAGGATGAAC AGTATATCCCCCCCTAGCAAGAAATATTGCTCATGCGGGGCCATCAGTAGACCTGGCAATTTTCTCACTCCACTTAGCTGGG GCATCCTCAATTTTGGGGGCCATTAACTTTATTACTACAGTAATTAATATGCGCTGGTCTGGACTACGTCTAGAGCGAATCCC GCTATTTGTATGAGCAGTAGTAATTACTGTCGTACTACTATTACTATCATTACCAGTTCTTGCCGGAGCTATTACAATACTAC TAACAGACCGAAACCTAAATACATCATTCTTCGACCCAGCGGGAGGGGGGGACCCAATTCTGTACCAACACCTATTTTGA mega BLAST - A. carnosus Identities=641/642 (99\%).

> WO46 "Drawida halla Jeju \#2 VI 2012" megaBLAST=Amynthas corticis (!). Redone as w24 (=Moniligastridae), i.e., sample "WO46” mixed in genetics lab maybe with WO47 that had nil result and was redone as w25 (or mixed with WO50) (see Blakemore, 2013a).

TATACTTCATTTTAGGAATTTGAGCCGGAATAATTGGGGCTGGAATAAGTCTTCTTATTCGAATCGAATTAAGACAACCTGG GTCATTCCTGGGAAATGACCAACTTTATAACACAATTGTAACAGCACATGCATTCTTAATAATTTTTTTTCTAGTAATACCAG TATTTATTGGTGGTTTTGGTAATTGACTATTACCACTTATATTGGGGACTCCAGATATAGCATTTCCACGCCTAAATAATATA AGATTCTGACTATTACCCCCCTCACTCATCTTATTAGTCTCTTCTGCAGCAGTGGAAAAAGGTGCAGGAACAGGATGAACAG TTTACCCCCCACTAGCAAGTAACATTGCGCATGCTGGACCATCAGTAGATCTGGCAATTTTCTCACTACACTTAGCAGGGGC ATCATCAATTCTAGGTGCAATCAATTTTATTACTACAGTAATTAATATACGATGATCAGGCCTACGACTAGAACGAATTCCC CTATTTGTATGAGCAGTAGTAATTACCGTAGTTCTACTACTTCTATCATTACCAGTACTAGCCGGTGCTATCACAATACTATT AACAGATCGAAACCTTAATACATCCTTCTTTGATCCAGCAGGAGGGGGAGACCCAATTTTATATCAACACC

$>$ WO56 Metaphire cf. agrestis from Cheonji - BLAST 100\% A. corticis - Mixed?

WO56 vs. w33 Identities $=646 / 646(100 \%)$ i.e., samples were mixed.

> WO67 Brown A. carnosus from Jeju bot gars 9/9/12 (see Blakemore, 2013a).

GGTCAACAAATCATAAAGATATTGGAACTTTATATTTTATCTTGGGAATCTGGGCAGGAATAATTGGTGCCGGTATGAGACT ACTTATTCGAATCGAGCTCAGGCAACCGGGCTCCTTTCTGGGAAGGGACCAACTATATAACACAATTGTAACAGCACACGC ATTCCTTATAATTTTCTTTCTAGTTATACCAGTATTCATTGGGGGATTTGGAAATTGGCTACTACCACTCATACTGGGAACAC CGGACATAGCATTTCCACGCCTAAACAACATGAGATTCTGGCTACTGCCCCCATCACTAATCTTACTAGTATCTTCCGCCGC AGTAGAGAAAGGAGCAGGTACAGGATGAACAGTATATCCCCCCCTAGCAAGAAATATTGCTCATGCGGGGCCATCAGTAGA 
CCTGGCAATTTTCTCACTCCACTTAGCTGGGGCATCCTCAATTTTGGGGGCCATTAACTTTATTACTACAGTAATTAATATGC GCTGGTCTGGACTACGTCTAGAGCGAATCCCGCTATTTGTATGAGCAGTAGTAATTACTGTCGTACTACTATTACTATCATTA CCAGTTCTTGCCGGAGCTATTACAATACTACTAACAGACCGAAACCTAAATACATCATTCTTCGACCCAGCGGGAGGGGGG GACCCAATTCTGTACCAACACCTATTTTGATTTTTTGGTCACCCTGAAGTTTA megaBLAST $99 \%$ A. carnosus Nara. nBLAST WO67 vs. WO66 Identities $=598 / 710$ (84\%). Also WO67 vs. WO24 (Identities=657/657 (100\%), WO67 vs. WO32 (Identities= 655/655 (100\%), WO67 vs. WO34 (Identities=657/657 (100\%) - i.e. all=A. carnosus .

> w25 "A. diffringens" Mt Halla temple (redo of WO47 that had nil result) IV250394. AACTCTATACTTCATTTTAGGAATTTGAGCCGGAATAATTGGGGCTGGAATAAGTCTTCTTATTCGAATCGAATTAAGACAA CCTGGGTCATTCCTGGGAAATGACCAACTTTATAACACAATTGTAACAGCACATGCATTCTTAATAATTTTTTTTCTAGTAAT ACCAGTATTTATTGGTGGTTTTGGTAATTGACTATTACCACTTATATTGGGGACTCCAGATATAGCATTTCCACGCCTAAATA ATATAAGATTCTGACTATTACCCCCCTCACTCATCTTATTAGTCTCTTCTGCAGCAGTGGAAAAAGGTGCAGGAACAGGATG AACAGTTTACCCCCCACTAGCAAGTAACATTGCGCATGCTGGACCATCAGTAGATCTGGCAATTTTCTCACTACACTTAGCA GGGGCATCATCAATTCTAGGTGCAATCAATTTTATTACTACAGTAATTAATATACGATGATCAGGCCTACGACTAGAACGAA TTCCCCTATTTGTATGAGCAGTAGTAATTACCGTAGTTCTACTACTTCTATCATTACCAGTACTAGCCGGTGCTATCACAATA CTATTAACAGATCGAAACCTTAATACATCCTTCTTTGATCCAGCAGGAGGGGGAGACCCAATTTTATATCAACACCTATTT megaBLAST Id=99-100\% A. corticis as expected. Unfigured and undissected specimen (seemingly mixed with WO46 Drawida initially).

>w31 A. cheyonjii (redo of WO55). BLAST 100\% "Amynthas gracilis" GenBank vouchers (AB542484.1 \& 542485.1) specimens were both from Ogasawara Islands, on Chichijima and Hahajima; whereas Identities=623/637 (98\%) for two specimens of Amynthas gracilis (AB542589.1 \& 542491.1) from Japanese mainland. Therefore, my conclusion was a new sub-species or species but cf. A. gracilis (see Blakemore, 2013a).

$>$ w33 (redo of WO57) A corticis saeseum Blakemore, 2013 Holotype - IV251310.

AACCCTATACTTCATTTTAGGAATTTGAGCCGGAATAATTGGGGCAGGAATAAGTCTTCTTATTCGAATCGAATTAAGACAA CCAGGATCATTCCTGGGAAGTGACCAACTATATAACACAATTGTAACAGCACATGCATTTTTAATAATTTTTTTTCTAGTAAT ACCAGTATTTATTGGTGGCTTTGGTAACTGATTATTACCACTTATATTAGGAACTCCAGATATAGCATTCCCACGCCTAAATA ATATAAGATTCTGACTATTGCCTCCATCGCTCATCCTATTAGTCTCTTCTGCAGCAGTAGAAAAGGGTGCGGGAACAGGATG AACAGTATATCCCCCACTAGCAAGTAATATTGCACATGCTGGACCATCAGTAGATCTAGCAATTTTCTCACTACACTTAGCG GGGGCATCATCAATTCTAGGTGCAATCAATTTTATTACTACAGTAATTAATATACGATGATCCGGGCTACAACTGGAACGAA TTCCCCTATTTGTATGAGCAGTGGTAATCACCACAGTTTTACTACTTATATCATTACCAGTACTAGCAGGTGCTATTACAATA CTATTAACAGATCGAAACCTTAATACATCATTCTTTGATCCTGCGGGAGGGGGGGACCCAATTTTATATCAACATCTATTT megaBLAST 99-100\% A. corticis from Japan that has note: "The organism belongs to Amynthas heteropoda, presently a junior synonym of Amynthas corticis".

This then is a allopatric and morphological sub-species that has recently diverged and is thus undetected by mitochondrial change (see Blakemore, 2013a).

nBLAST w33 vs. w25 (redo of WO47) Id=614/658 (93\%) i.e., potentially different spp.

nBLAST w33 vs. w59 A. corticis from Incheon, $\mathrm{Id}=611 / 654$ (93\%), i.e., ditto.

>w37 A. carnosus from Geoman, Korea (RJB - see Blakemore, 2012a; 2013c).

AACTTTATATTTTATCTTGGGAATCTGGGCAGGAATAATTGGTGCCGGTATGAGACTACTTATTCGAATCGAGCTCAGGCAA CCGGGCTCCTTTCTGGGAAGGGACCAACTATATAACACAATTGTAACAGCACACGCATTCCTTATAATTTTCTTTCTAGTTAT ACCAGTATTCATTGGGGGATTTGGAAATTGGCTACTACCACTCATACTGGGAACACCGGACATAGCATTTCCACGCCTAAAC AACATGAGATTCTGGCTACTGCCCCCATCACTAATCTTACTAGTATCTTCCGCCGCAGTAGAGAAAGGAGCAGGTACAGGAT GAACAGTATATCCCCCCCTAGCAAGAAATATTGCTCATGCGGGGCCATCAGTAGACCTGGCAATTTTCTCACTCCACTTAGC TGGGGCATCCTCAATTTTGGGGGCCACTAACTTTATTACTACAGTAATTAATATGCGCTGGTCTGGACTACGTCTAGAGCGA ATCCCGCTATTTGTATGAGCAGTAGTAATTACTGTCATACTACTATTACTATCATTACCAGTTCTTGCCGGAGCTATTACAAT ACTACTAACAGACCGAAACCTAAATACATCATTCTTCGACCCAGCGGGAGGGGGGGACCCAATTCTGTACCAACACCTATTT megaBLAST Maximum Identity=99\% A. carnosus (AB543184.1) from Nara, Japan.

> w54 Amynthas carnosus naribunji sp. nov. H from Ullungdo (cf Blakemore, 2013b).

TTATACTTTATCTTAGGAATCTGAGCCGGAATAATTGGTGCTGGTATAAGACTTCTTATTCGAATTGAGCTCAGACAACCAG GATCCTTTCTGGGAAGAGATCAACTATATAACACAATTGTAACAGCACACGCATTCCTGATAATTTTCTTTCTAGTTATACCC GTATTCATTGGGGGATTTGGAAATTGACTACTACCACTTATACTAGGAACACCGGATATAGCATTTCCGCGCCTAAACAATA TGAGATTTTGACTATTACCACCATCACTAATCTTACTAGTCTCTTCAGCCGCAGTAGAGAAAGGAGCGGGGACAGGATGGAC AGTATATCCCCCCCTAGCAAGAAATATTGCTCATGCAGGGCCATCAGTAGACCTAGCAATTTTCTCACTCCACTTAGCTGGA 
GCATCCTCAATTTTAGGGGCCATTAACTTCATTACTACAGTAATTAATATGCGCTGGTCTGGACTACGCCTAGAGCGAATTC CACTATTTGTGTGAGCTGTAGTAATTACTGTGGTGCTACTATTACTATCGTTACCAGTTCTTGCCGGAGCTATTACAATACTA CTAACAGACCGAAACTTAAATACATCATTCTTCGACCCAGCGGGAGGGGGAGACCCAATTTTATACCAACATCTATTT megaBLAST Maximum Identity $=93 \%$ A. carnosus (AB543184.1) from Nara, Japan. nBLAST > w54 vs. w56 A. carnosus roki $\mathrm{H}$ from Incheon. Identities $=607 / 652(93 \%)$. nBLAST > w54 vs. w37 A. carnosus carnosus from Geoman. Identities=603/654 (92\%).

w56 and w57 see Blakemore and Lee (2013 this issue).

> w59 Incheon A. corticis? (RJB) - NIBRIV261267. ACTCTATACTTCATTTTAGGAATTTGAGCCGGAATAATTGGGGCTGGAATAAGTCTTCTTATTCGAATCGAATTAAGACAAC CTGGGTCATTCCTGGGAAGTGACCAACTTTATAACACAATTGTAACAGCACATGCATTCTTAATAATTTTTTTTCTAGTAATA CCAGTATTTATTGGTGGTTTTGGTAATTGACTATTACCACTTATATTGGGGACTCCAGATATAGCATTTCCACGCCTAAATAA TATAAGATTCTGACTATTACCCCCCTCACTCATCTTACTAGTCTCTTCTGCAGCAGTGGAAAAAGGTGCAGGAACAGGATGA ACAGTTTACCCACCACTAGCAAGTAACATTGCGCATGCTGGACCATCAGTAGATCTGGCAATTTTCTCACTACACTTAGCAG GGGCATCATCAATTCTAGGTGCAATCAATTTTATTACTACAGTAATTAATATACGATGATCAGGCCTACGACTAGAACGAAT TCCCCTATTTGTATGAGCAGTAGTAATTACCGTAGTTCTACTACTTCTATCATTACCAGTACTAGCCGGTGCTATCACAATAC TATTAACAGATCGAAACCTTAATACATCATTCTTTGATCCAGCAGGAGGGGGAGACCCAATTTTATATCAACACCTATTTTG megaBLAST Id=100\% Amynthas corticis voucher(DQ224190) from Taiwan.

> w63 Amynthas gracilis Cheonji, Jeju 12 June 2012 - (see Blakemore, 2013a).

TATATTTTATTCTAGGAATTTGAGCCGGAATAATTGGAGCTGGAATAAGGCTACTTATTCGAATTGAACTCAGACAGCCGGG ATCGTTTCTGGGAAGAGATCAACTATATAATACAATTGTAACAGCTCATGCATTTGTAATAATTTTCTTTCTGGTAATACCAG TATTCATTGGTGGATTTGGAAACTGACTACTACCTCTAATGCTGGGTACACCAGACATAGCATTTCCGCGACTTAATAATAT AAGATTTTGACTACTCCCCCCGTCACTTATCTTACTAGTAAGATCCGCGGCCGTTGAAAAGGGGGCGGGAACTGGATGAACA GTATATCCCCCGCTGGCAAGAAATATTGCACATGCTGGTCCATCAGTAGATCTAGCAATCTTCTCACTACACTTGGCAGGAG CATCATCTATTCTTGGGGCCATTAACTTTATTACAACTGTAATTAATATACGATGATCTGGATTACGGCTAGAACGAATCCCC CTATTTGTATGGGCCGTAGTAATTACTGTAGTACTTCTACTATTATCTCTACCTGTACTAGCCGGAGCTATTACTATATTATTA ACAGATCGAAACCTTAACACATCATTCTTTGATCCCGCTGGAGGTGGAGACCCTATTCTATATCAACACCTA megaBLAST Id=100\% Amynthas gracilis (AB542485.1) from Japan.

nBLAST $w 63$ vs. WO54 Identities $=650 / 650(100 \%)$, i.e., WO54 was mixed.

nBLAST w63 vs. w31 Identities $=650 / 650(100 \%)$ - QED.

> JET112-115IAn-460-461। Hikone, Shiga, Japan, initial id as A. cf. pingi (=A. carnosus).

AACTTTATATTTTATCTTGGGAATCTGGGCAGGAATAATTGGTGCCGGTATGAGACTACTTATTCGAATCGAGCTCAGGCAA CCGGGCTCCTTTCTGGGAAGGGACCAACTATATAACACAATTGTAACAGCACACGCATTCCTTATAATTTTCTTTCTAGTTAT ACCAGTATTCATTGGGGGATTTGGAAATTGGCTACTACCACTCATACTGGGAACACCGGACATAGCATTTCCACGCCTAAAC AACATGAGATTCTGGCTACTGCCCCCATCACTAATCTTACTAGTATCTTCCGCCGCAGTAGAGAAAGGAGCAGGTACAGGAT GAACAGTATATCCCCCCCTAGCAAGAAATATTGCTCATGCGGGGCCATCAGTAGACCTGGCAATTTTCTCACTCCACTTAGC TGGGGCATCCTCAATTTTGGGGGCCATTAACTTTATTACTACAGTAATTAATATGCGCTGGTCTGGACTACGTCTAGAGCGA ATCCCGCTATTTGTATGAGCAGTAGTAATTACTGTCGTACTACTATTACTATCATTACCAGTTCTTGCCGGAGCTATTACAAT ACTACTAACAGACCGAAACCTAAATACATCATTCTTCGACCCAGCGGGAGGGGGGGACCCAATTCTGTACCAACACCTATTT megaBLAST Maximum Identity=99\% A. carnosus (AB543184.1) from Nara, Japan.

> JET120 Amynthas cf. corticis Shiga Japan (Tokyo An-464 RJB).

AACCCTATACTTCATTTTAGGAATTTGAGCCGGAATAATTGGGGCAGGAATAAGTCTTCTTATTCGAATCGAATTAAGACAA CCAGGATCATTCCTGGGAAGTGACCAACTATATAACACAATTGTAACAGCACATGCATTTTTAATAATTTTTTTTCTAGTAAT ACCAGTATTTATTGGTGGCTTTGGTAACTGATTATTACCACTTATATTAGGAACTCCAGATATAGCATTCCCACGCCTAAATA ATATAAGATTCTGACTATTGCCTCCATCGCTCATCCTATTAGTCTCTTCTGCAGCAGTAGAAAAGGGTGCGGGAACAGGATG AACAGTATATCCCCCACTAGCAAGTAATATTGCACATGCTGGACCATCAGTAGATCTAGCAATTTTCTCACTACACTTAGCG GGGGCATCATCAATTCTAGGTGCAATCAATTTTATTACTACAGTAATTAATATACGATGATCCGGGCTACAACTGGAACGAA TTCCCCTATTTGTATGAGCAGTGGTAATCACCACAGTTTTACTACTTATATCATTACCAGTACTAGCAGGTGCTATTACAATA CTATTAACAGATCGAAACCTTAATACATCATTCTTTGATCCTGCGGGAGGGGGGGACCCAATTTTATATCAACATCTATTT

> JET122 Amynthas cf. corticis Biwa Shiga Japan (Tokyo An-465 RJB).

AACTCTATACTTCATTTTAGGAATTTGAGCCGGAATAATTGGGGCTGGAATAAGTCTTCTTATTCGAATCGAATTAAGACAA CCTGGGTCATTCCTGGGAAGTGACCAACTTTATAACACAATTGTAACAGCACATGCATTCTTAATAATTTTTTTTCTAGTAAT 
ACCAGTATTTATTGGTGGTTTTGGTAATTGACTATTACCACTTATATTGGGGACTCCAGATATAGCATTTCCACGCCTAAATA ATATAAGATTCTGACTATTACCCCCCTCACTCATCTTACTAGTCTCTTCTGCAGCAGTGGAAAAAGGTGCAGGAACAGGATG AACAGTTTACCCACCACTAGCAAGTAACATTGCGCATGCTGGACCATCAGTAGATCTGGCAATTTTCTCACTACACTTAGCA GGGGCATCATCAATTCTAGGTGCAATCAATTTTATTACTACAGTAATTAATATACGATGATCAGGCCTACGACTAGAACGAA TTCCCCTATTTGTATGAGCAGTAGTAATTACCGTAGTTCTACTACTTCTATCATTACCAGTACTAGCCGGTGCTATCACAATA CTATTAACAGATCGAAACCTTAATACATCATTCTTTGATCCAGCAGGAGGGGGAGACCCAATTTTATATCAACACCTATTT

> JET138 Amynthas sp. Mishima Japan (Tokyo An-473 RJB).

AACCCTATACTTCATTTTAGGAATTTGAGCCGGAATAATTGGGGCAGGAATAAGTCTTCTTATTCGAATCGAATTAAGACAA CCAGGATCATTCCTGGGAAGTGACCAACTATATAACACAATTGTAACAGCACATGCATTTTTAATAATTTTTTTTCTAGTAAT ACCAGTATTTATTGGTGGCTTTGGTAACTGATTATTACCACTTATATTAGGAACTCCAGATATAGCATTCCCACGCCTAAATA ATATAAGATTCTGACTATTGCCTCCATCGCTCATCCTATTAGTCTCTTCTGCAGCAGTAGAAAAGGGTGCGGGAACAGGATG AACAGTATATCCCCCACTAGCAAGTAATATTGCACATGCTGGACCATCAGTAGATCTAGCAATTTTCTCACTACACTTAGCG GGGGCATCATCAATTCTAGGTGCAATCAATTTTATTACTACAGTAATTAATATACGATGATCCGGGCTACAACTGGAACGAA TTCCCCTATTTGTATGAGCAGTGGTAATCACCACAGTTTTACTACTTATATCATTACCAGTACTAGCAGGTGCTATTACAATA CTATTAACAGATCGAAACCTTAATACATCATTCTTTGATCCTGCGGGAGGGGGGGACCCAATTTTATATCAACATCTATTT

> JET140 Amynthas cf. heteropodus Mishima Japan (Tokyo An-474 RJB).

AACTCTATACTTCATTTTAGGAATTTGAGCCGGAATAATTGGGGCTGGAATAAGTCTTCTTATTCGAATCGAATTAAGACAA CCTGGGTCATTCCTGGGAAGTGACCAACTTTATAACACAATTGTAACAGCACATGCATTCTTAATAATTTTTTTTCTAGTAAT ACCAGTATTTATTGGTGGTTTTGGTAATTGACTATTACCACTTATATTGGGGACTCCAGATATAGCATTTCCACGCCTAAATA ATATAAGATTCTGACTATTACCCCCCTCACTCATCTTACTAGTCTCTTCTGCAGCAGTGGAAAAAGGTGCAGGAACAGGATG AACAGTTTACCCACCACTAGCAAGTAACATTGCGCATGCTGGACCATCAGTAGATCTGGCAATTTTCTCACTACACTTAGCA GGGGCATCATCAATTCTAGGTGCAATCAATTTTATTACTACAGTAATTAATATACGATGATCAGGCCTACGACTAGAACGAA TTCCCCTATTTGTATGAGCAGTAGTAATTACCGTAGTTCTACTACTTCTATCATTACCAGTACTAGCCGGTGCTATCACAATA CTATTAACAGATCGAAACCTTAATACATCATTCTTTGATCCAGCAGGAGGGGGAGACCCAATTTTATATCAACACCTATTT

> JET142 Amynthas sp. Ueno Tokyo Japan (Tokyo An-475 RJB).

AACCCTATATTTTATTCTAGGAATTTGAGCCGGAATAATTGGAGCTGGAATAAGGCTACTTATTCGAATTGAACTCAGACAG CCGGGATCGTTTCTGGGAAGAGATCAACTATATAATACAATTGTAACAGCTCATGCATTTGTAATAATTTTCTTTCTGGTAAT ACCAGTATTCATTGGTGGATTTGGAAACTGACTACTACCTCTAATGCTGGGTACACCAGACATAGCATTTCCGCGACTTAAT AATATAAGATTTTGACTACTCCCCCCGTCACTTATCTTACTAGTAAGATCCGCGGCCGTTGAAAAGGGGGCGGGAACTGGAT GAACAGTATATCCCCCGCTGGCAAGAAATATTGCACATGCTGGTCCATCAGTAGATCTAGCAATCTTCTCACTACACTTGGC AGGAGCATCATCTATTCTTGGGGCCATTAACTTTATTACAACTGTAATTAATATACGATGATCTGGATTACGGCTAGAACGA ATCCCCCTATTTGTATGGGCCGTAGTAATTACTGTAGTACTTCTACTATTATCTCTACCTGTACTAGCCGGAGCTATTACTAT ATTATTAACAGATCGAAACCTTAACACATCATTCTTTGATCCCGCTGGAGGTGGAGACCCTATTCTATATCAACACCTATTT megaBLAST id=100\% AB542485 “Amynthas gracilis" voucher, from Ogasawara Islands, Chichijima.

megaBLAST id=99\% JX069935 “Amynthas sp.” from Hainan.

> JET153 Amynthas cf. corticis Tamworth NSW Australia (Tokyo An-477.1 RJB).

AACCCTATATTTTATTCTAGGAATTTGAGCCGGAATAATTGGAGCTGGAATAAGGCTACTTATTCGAATTGAACTCAGACAG CCGGGATCGTTTCTGGGAAGAGATCAACTATATAATACAATTGTAACAGCTCATGCATTTGTAATAATTTTCTTTCTGGTAAT ACCAGTATTCATTGGTGGATTTGGAAACTGACTACTACCTCTAATGCTGGGTACACCAGACATAGCATTTCCGCGACTTAAT AATATAAGATTTTGACTACTCCCCCCGTCACTTATCTTACTAGTAAGATCCGCGGCCGTTGAAAAGGGGGCGGGAACTGGAT GAACAGTATATCCCCCGCTGGCAAGAAATATTGCACATGCTGGTCCATCAGTAGATCTAGCAATCTTCTCACTACACTTGGC AGGAGCATCATCTATTCTTGGGGCCATTAACTTTATTACAACTGTAATTAATATACGATGATCTGGATTACGGCTAGAACGA ATCCCCCTATTTGTATGGGCCGTAGTAATTACTGTAGTACTTCTACTATTATCTCTACCTGTACTAGCCGGAGCTATTACTAT ATTATTAACAGATCGAAACCTTAACACATCATTCTTTGATCCCGCTGGAGGTGGAGACCCTATTCTATATCAACACCTATTT

> JET154 Amynthas cf. corticis Tamworth NSW Australia (Tokyo An-477.2 RJB).

AACCCTATATTTTATTCTAGGAATTTGAGCCGGAATAATTGGAGCAGGAATAAGACTACTTATTCGAATTGAGCTCAGACAG CCGGGATCGTTTCTGGGAAGAGATCAATTATATAATACAATTGTAACAGCTCATGCATTCGTAATAATTTTCTTTCTAGTAAT ACCAGTATTCATTGGTGGATTTGGAAACTGACTACTACCTCTAATGCTGGGTACACCAGACATAGCATTTCCGCGGCTTAAT AATATAAGATTTTGGCTACTCCCCCCGTCACTTATCTTACTAGTAAGATCCGCGGCCGTTGAAAAGGGTGCGGGAACTGGAT GGACAGTATATCCCCCGCTGGCAAGAAATATTGCACATGCTGGTCCATCAGTAGATCTAGCAATCTTCTCACTACACTTAGC AGGGGCATCATCTATTCTTGGGGCCATTAACTTTATTACAACTGTAATTAATATGCGATGATCTGGATTACGGCTAGAGCGA ATCCCCCTATTTGTATGGGCCGTAGTAATTACTGTAGTACTTCTACTATTATCTCTACCTGTACTAGCCGGAGCTATTACTAT ACTATTAACAGATCGAAACCTTAACACATCATTCTTTGATCCCGCTGGAGGTGGAGACCCTATTCTATATCAACACCTATTT 
JET153 +JET154 - were from same specimen and should be same but results differed slightly $(<1 \%)$.

$>$ GenBank AB543184I Amynthas carnosus voucher from Nara, Japan.

> GenBank DQ224190l Amynthas corticis voucher from Taiwan.

> GenBank DQ257319 “Amynthas diffringens" voucher from East Texas.

$>$ GenBank EF077550| "Amynthas diffringens" voucher from China.

> GenBank EF077572 “Amynthas homochaetus" voucher from China.

> GenBank EF077559l “Amynthas heterochaetus" voucher from China.

> GenBank JX290433 “Amynthas corticis” voucher from Taiwan.

Amynthas penpuensis Shen et al., 2003 DNA data not yet on Genbank (July, 2013).

Amynthas taiwumontis Shen et al., 2013 DNA data yet to be reported(July, 2013). 